December 2021

"Keeping the Agents in the Dark: Private Disclosures in Competing Mechanisms"

Andrea Attar, Eloisa Campioni, Thomas Mariotti and Alessandro Pavan 


\title{
Keeping the Agents in the Dark: Private Disclosures in Competing Mechanisms*
}

\author{
Andrea Attar ${ }^{\dagger}$ \\ Thomas Mariotti ${ }^{\S}$ \\ Eloisa Campioni ${ }^{\ddagger}$ \\ Alessandro Pavan $\mathbb{I}$
}

December 13, 2021

\begin{abstract}
We study competing-mechanism games, in which several principals contract with several privately informed agents. We show that enabling the principals to engage into private disclosures - whereby a principal sends to the agents contractible private signals about how her final decision will respond to the agents' messages - can significantly affect the predictions of such games. Our first result is that equilibrium outcomes and payoffs of games without private disclosures may not be supported when private disclosures are allowed for. This challenges the robustness of the folk theorems à la Yamashita (2010). Our second result is that allowing for private disclosures may generate equilibrium outcomes and payoffs that cannot be supported in any game without private disclosures, no matter how rich the message spaces are. This challenges the canonicity of the universal mechanisms of Epstein and Peters (1999). Together, these findings call for a novel approach to the analysis of competing-mechanism games.
\end{abstract}

Keywords: Incomplete Information, Competing Mechanisms, Private Disclosures, Folk Theorems, Universal Mechanisms.

JEL Classification: D82.

${ }^{*}$ We thank Laura Doval, Piotr Dworczak, Johannes Hörner, Michael Peters, Martin Pollrich, and Takuro Yamashita for extremely valuable feedback. We also thank seminar audiences at Brown University, EIEF, Higher School of Economics, LUISS, New Economic School, Northwestern University, Universität Bonn, Université de Montréal, University of British Columbia, UCLA, UC Riverside, and the Virtual Market Design Seminar, as well as conference participants at the 2020 Econometric Society World Congress, the 2021 ASSA Virtual Annual Meeting, the 2021 CEPR Workshop on Contracts, Incentives, and Information, and the 2021 Università degli Studi di Roma "Tor Vergata" Workshop on Markets, Information, and the Macroeconomy for many useful discussions. This research has benefited from financial support of the ANR (Programme d'Investissements d'Avenir ANR-17-EURE-0010), the MIUR (PRIN 2015), and the research foundation TSE-Partnership.

†Toulouse School of Economics, CNRS, University of Toulouse Capitole, Toulouse, France, Università degli Studi di Roma "Tor Vergata," Roma, Italy, and CESifo. Email: andrea.attar@tse-fr.eu.

‡Università degli Studi di Roma "Tor Vergata," Roma, Italy. Email: eloisa.campioni@uniroma2.it.

$\S$ Toulouse School of Economics, CNRS, University of Toulouse Capitole, Toulouse, France, CEPR, and CESifo. Email: thomas.mariotti@tse-fr.eu.

INorthwestern University, Evanston, United States, and CEPR. Email: alepavan@northwestern.edu. 


\section{Introduction}

Classical mechanism-design theory identifies the holding of private information by economic agents as a fundamental constraint on the allocations of resources (Hurwicz (1973)). How agents communicate their private information then becomes crucial for determining the set of allocations that can be implemented. In pure incomplete-information environments, in which all payoff-relevant decisions are taken by a single uninformed principal, one can with no loss of generality restrict all private communication to be one-sided, from the agents to the principal (Myerson (1979)). Indeed, in that case, the principal need only post a mechanism selecting a (possibly random) decision for every profile of messages she may receive from the agents - what we hereafter refer to as a standard mechanism. Communication from the principal to the agents is then limited to the public announcement of such a mechanism; private communication from the principal to the agents is redundant, as it has no bearing on the set of allocations that the principal can implement.

In this paper, we argue that these basic insights from classical mechanism-design theory do not extend to competitive settings. To this end, we consider competing-mechanism games, in which the implementation of an allocation is no longer in the hands of a single principal, but of several principals who noncooperatively design mechanisms, each of which controls a specific dimension of the allocation. In this context, we show that allowing for private communication from the principals to the agents can significantly affect the set of allocations that can be supported in an equilibrium of such a game, and this, even if one focuses, as we do, on pure incomplete-information environments in which agents take no payoff-relevant decisions - arguably the least favorable scenario for this form of private communication to have bite. The general lesson from our results is that, in spite of their universal use in the theoretical and applied literature, the restriction to standard mechanisms is unwarranted in competitive settings, which calls for a drastic shift in the conceptualization and analysis of competing-mechanism games.

To put our findings in perspective, it is useful to keep in mind the two theoretical pillars of the competing-mechanism literature. First, following Epstein and Peters (1999), one can construct a space of (indirect) universal mechanisms, whereby each agent can communicate to each principal his endogenous market information - that is, the profile of mechanisms posted by the other principals - in addition to his exogenous private information. An analog of the revelation principle holds: any equilibrium outcome of any competing-mechanism game can be supported as an equilibrium outcome of the game in which the principals can only post universal mechanisms. This addresses the infinite-regress problem raised by the failure 
of the standard revelation principle in competing-mechanism games (McAfee (1993), Peck (1997)). Second, following Yamashita (2010), one can obtain an explicit characterization of equilibrium outcomes and equilibrium payoffs for a large class of competing-mechanism games. An analog of the folk theorem holds: any incentive-compatible allocation that yields each principal a payoff above a well-defined min-max-min bound can be supported in equilibrium by each principal posting a recommendation mechanism, whereby the agents, in addition to reporting their exogenous private information, are asked to vote on the direct mechanism the principal should use. This, in turn, enables them to implement punishments following any deviation by some other principal.

From our perspective, the key point is that these two central results are established under the assumption that principals are restricted to post standard mechanisms, so that all private communication is from the agents to the principals. In particular, both universal and recommendation mechanisms are instances of standard mechanisms. We challenge these results by considering a richer class of communication protocols in which the principals can privately disclose information to the agents.

We model private disclosures from the principals to the agents as contractible private signals sent by each principal to the agents about her decision rule, that is, the mapping describing how the decision she implements responds to the messages she receives from the agents. These private signals are sent to the agents before the latter have the opportunity to send messages to the principals. Each principal fully commits, as parts of the mechanism she posts, to a distribution of private signals and to a decision rule mapping the private signals she initially sends to the agents and the messages she ultimately receives from them into a (possibly random) decision. Notice that the extension of the game we thereby propose has no bite in the case of a single principal who can control all the dimensions of the allocation, as private disclosures do not affect the set of allocations that she can implement. In practice, such private disclosures may take the form of sellers privately informing buyers in an auction of personalized reservation prices before they submit their bids, manufacturers privately informing retailers of the amount of output supplied, or policy makers privately informing voters of the characteristics of a public good implemented as a result of the solicitation of the voters' preferences.

Our first result is that equilibria in standard mechanisms need not be robust to private disclosures. To establish this result, we start from an example of a competing-mechanism game without private disclosures in which message spaces are sufficiently rich for the two principals in the game to post recommendation mechanisms. In this game, any feasible 
payoff vector for the principals can be supported in equilibrium. ${ }^{1}$ However, we show that, by posting a mechanism that asymmetrically discloses information about her decisions to the agents, one of the principals can ensure that the agents no longer have the incentives to carry out the punishment in the other principal's mechanism that would be necessary to make the deviation unprofitable. Intuitively, this is because, by privately informing one of the agents of her decision while keeping the other agents in the dark, this principal is able to perfectly align her preferences in each state with those of that agent, making the latter no longer willing to participate in the required punishment. As a result, this principal can guarantee herself a payoff strictly above her minimum feasible payoff, regardless of the mechanism posted by the other principal and of the continuation equilibrium played by the agents. Many equilibrium payoffs that can be supported when principals compete in standard mechanisms can thus no longer be supported when, in addition to standard mechanisms, principals can also post mechanisms with private signals. The upshot of this example is that equilibrium outcomes and payoffs of competing-mechanism games without private disclosures, and, in particular, those supported by recommendation mechanisms as in the folk theorem of Yamashita (2010), need not be supported when principals have the opportunity to engage into private disclosures.

Our second result is that equilibrium outcomes and payoffs of competing-mechanism games with private disclosures need not be supported in any game in which principals are restricted to post standard mechanisms, no matter how rich the message spaces are. The reason is that private disclosures may help the principals correlate their decisions with the agents' exogenous private information in a way that cannot be replicated by principals responding to the agents' messages when these are solely based on the agents' common knowledge of the mechanisms and on their exogenous private information. To establish this result, we provide an example of a competing-mechanism game with private disclosures in which the equilibrium correlation between the principals' decisions and the agents' exogenous private information requires that (1) the agents receive information about one principal's decision and pass it on to the other principal before the latter finalizes her own decision, and (2) such information not create common knowledge among the agents about the former principal's decision before they communicate with the latter. The example illustrates the possibility to achieve both (1) and (2) with private disclosures, and the necessity of both (1) and (2) when it comes to supporting certain outcomes and payoffs, which implies the impossibility of supporting these with standard mechanisms, no matter how rich the message

\footnotetext{
${ }^{1}$ This reflects that, in the example, the min-max-min bound computed over recommendation mechanisms is equal to the minimum feasible payoff for each principal.
} 
spaces are. In equilibrium, the requirement (2) is satisfied by letting one principal send private signals, each of which is completely uninformative of the principal's decision from any agent's perspective, but which together, once they are passed on by the agents to the other principal in an incentive-compatible way, perfectly reveal the principal's decision; thus private disclosures in this example play the role of an encrypted message that one principal passes on to the other through the agents, while keeping the latter in the dark. The upshot of this example is that the universal mechanisms of Epstein and Peters (1999) are no longer canonical when principals can engage into private disclosures.

Taken together, the above results imply that the sets of equilibrium outcomes and payoffs of competing-mechanism games with and without private disclosures are not nested. These results challenge the existing modeling approaches and suggest that private disclosures from the principals to the agents should be central to the theory of competing mechanisms.

\section{Related Literature}

This paper contributes to the theoretical foundations of competing-mechanism games. In a seminal paper, McAfee (1993) points out that the equilibria of such games may require that agents report all their private information to the principals; that is, their exogenous information about their private types and their market information about the mechanisms posted by the other principals. To overcome the resulting infinite-regress problem, Epstein and Peters (1999) construct a space of universal mechanisms that enables them to establish an analog of the revelation principle for competing-mechanism games. Subsequent work provides explicit characterizations of the equilibrium outcomes of such games. Yamashita (2010) shows that, if there are at least three agents, every deterministic incentive-compatible allocation yielding each principal a payoff at least equal to a well-defined min-max-min bound can be supported in equilibrium. Our results indicate that this characterization is sensitive to the assumption that the principals are restricted to standard mechanisms and does not extend when principals can engage into private disclosures. Peters and Troncoso-Valverde (2013), in a different context, argue that any allocation that is incentive-compatible and individually rational in the sense of Myerson (1979) can be supported in equilibrium provided there are sufficiently many players - there is no distinction between principals and agents in their setup. The distinctive feature of their modeling approach is that each player commits to a mechanism and to irreversibly sending an encrypted message about her type before observing the mechanisms posted by the other players and privately communicating with them. Each player, in particular, sends her encrypted type before knowing whether or not she 
will have to participate in punishing some other player, which allows for harsh punishments that are not incentive-compatible once the mechanisms are observed. By contrast, our approach fits more squarely into classical mechanism-design theory by maintaining the standard distinction between principals and agents, as well as the standard informational assumption that agents do not communicate among themselves and release no information before observing the mechanisms posted by the principals and the signals they individually receive from them.

In classical mechanism-design theory (Myerson (1982)), private communication from a single principal to the agents is key when certain payoff-relevant actions can solely be taken by the agents, as in moral-hazard settings. Such communication, taking the form of action recommendations to the agents, has been shown to serve as a correlating device between the players' decisions in several problems of economic interest, such as Rahman and Obara's (2010) model of partnerships. Perhaps surprisingly, however, private disclosures have been neglected in competing-mechanism settings even when agents take payoff-relevant actions, as in Prat and Rustichini's (2003) model of lobbying. To the best of our knowledge, the only exception is the recent work of Attar, Campioni, and Piaser (2019), who study complete-information games in which agents take observable actions. They construct an example in which equilibrium allocations supported by standard mechanisms fail to be robust against a deviation by a principal to a mechanism with private recommendations. In equilibrium, principals correlate their decisions with the agents' actions in a way that cannot be achieved without private recommendations.

Whereas private signals from the principals to the agents can still be interpreted, in the above papers, in light of the traditional role they play in single-principal settings, the present paper uncovers two novel roles for such signals, making them very different from action recommendations. Private signals first alleviate the punishments that can be inflicted on a principal. In our first example, disclosing her decision to one agent while keeping the other agents in the dark enables one principal to guarantee herself a payoff strictly above her min-max-min bound by making it impossible for the agents to coordinate on the required punishment. Private signals also help overcoming the lack of a direct communication channel between the principals. In our second example, one principal can correlate her decisions with those of another principal and with the agents' exogenous private information by sending signals to the agents, to be passed on to the other principal, in a way that cannot be replicated with standard mechanisms.

These features of private disclosures in our model appear particularly relevant for the 
applications of competing-mechanism games favored in the literature, such as competing auctions (McAfee (1993), Peters (1997), Peters and Severinov (1997), Virág (2010)) or competitive search (Moen (1997), Eeckhout and Kircher (2010), Wright, Kircher, Julien and Guerrieri (2021)). In these settings, contracting is typically decentralized - so that principals may find it difficult to rely on a common mediator to coordinate their decisions - and direct communication among the principals - either in the form of principals exchanging cheap-talk messages, or in the form of principals using semi-private correlation devices whose realizations are observed by the other principals but not by the agents at the time they communicate with the principals - is unlikely to be feasible.

In our setting, the correlation in the principals' decisions is generated by the agents communicating to each principal both their exogenous private information and the private signals they receive from other principals. The principals cannot directly condition on other principals' decisions and/or mechanisms, or directly exchange information among themselves. Instead, Kalai, Kalai, Lehrer and Samet (2010), Peters and Szentes (2012), Peters (2015), and Szentes (2015) consider settings in which players can make commitments contingent on other players' commitments and communication is unrestricted. The result in our first example extends to these settings: by deviating to a mechanism with private disclosures, a principal can guarantee herself a payoff strictly above her min-max-min bound, regardless of whether or not principals can make commitments contingent on other principals' decisions and/or mechanisms. The result in our second example, instead, extends to settings in which principals can condition their mechanism on other principals' mechanisms, as in Peters' (2015) model of reciprocal contracting, but not to settings in which principals can directly condition their decisions on other principals' decisions. This is because private disclosures in this example take the form of an encrypted message passed on from one principal to the other through the agents in an incentive-compatible way, something which can be achieved in a more direct way if principals can communicate directly among themselves after receiving the agents' messages.

The role of private signals we highlight in this paper hinges on at least two principals contracting with at least two agents. When there is a single principal, the revelation principle obviously holds and private disclosures have no bearing on the set of equilibrium outcomes. Similarly, when multiple principals contract with a single agent, and irrespective of whether contracting is simultaneous or sequential, the menu theorems of Peters (2001), Martimort and Stole (2002), and Pavan and Calzolari (2009, 2010) guarantee that any equilibrium outcome of a game in which the principals compete by offering arbitrary message-contingent 
decision rules can be reproduced in a game in which the principals offer subsets (menus) of their decisions to the agent and delegate to the latter the choice of the final allocation. Thus private disclosures play no role in such settings either.

In a collusion setting à la Laffont and Martimort (1997), von Negenborn and Pollrich (2020) show that a principal can prevent collusion between an agent and a supervisor by informing these two players asymmetrically of the decision she will take in response to their reports. In their model, the benefits of private disclosures disappear when the agent and the supervisor can write contracts conditioning their side payments on the principal's final decision. Instead, we focus on settings with competing principals, and show that the benefits of private disclosures remain even when principals can condition their decisions on the other principals' decisions. Most importantly, we establish that private disclosures undermine folk-theorem results and the construction of universal mechanisms established in the competing-mechanism literature.

The paper is organized as follows. Section 2 introduces a general model of competing mechanisms under incomplete information. Sections 3 and 4 present the results. Section 5 discusses the different roles of private disclosures, and the robustness of the results to alternative contracting assumptions and to the availability of public correlating devices. Section 6 concludes.

\section{The Model}

We consider a pure incomplete-information setting in which several principals, indexed by $j=1, \ldots, J$, contract with several agents, indexed by $i=1, \ldots, I$. As anticipated in the introduction, our results crucially hinge on $I \geq 2$ and $J \geq 2$.

Information Every agent $i$ (he) possesses some exogenous private information summarized by his type $\omega^{i}$, which belongs to some finite set $\Omega^{i}$. Thus the set of exogenous states of the world $\omega \equiv\left(\omega^{1}, \ldots, \omega^{I}\right)$ is $\Omega \equiv \Omega^{1} \times \ldots \times \Omega^{I}$. Principals and agents commonly believe that the state $\omega$ is drawn from $\Omega$ according to the distribution $\mathbf{P}$.

Decisions and Payoffs Every principal $j$ (she) takes a decision $x_{j}$ in some finite set $X_{j}$. We let $v_{j}: X \times \Omega \rightarrow \mathbb{R}$ and $u^{i}: X \times \Omega \rightarrow \mathbb{R}$ be the payoff functions of principal $j$ and of agent $i$, respectively, where $X \equiv X_{1} \times \ldots \times X_{J}$ is the set of possible profiles of decisions for the principals. Agents take no payoff-relevant decisions. An allocation is a function $z: \Omega \rightarrow \Delta(X)$ assigning a lottery over the set $X$ to every state of the world. The outcome 
induced by an allocation $z$ is the restriction of $z$ to the set of states occurring with positive probability under $\mathbf{P}^{2}$

Mechanisms with Signals A principal can engage into private disclosures by posting a mechanism with signals. Such a mechanism consists, first, of a probability distribution over the signals that the principal privately sends to the agents, and, second, of a decision rule that assigns a lottery over her decisions to every profile of signals she sends to the agents and every profile of messages she receives from them. Formally, a mechanism with signals for principal $j$ is a pair $\gamma_{j} \equiv\left(\sigma_{j}, \phi_{j}\right)$ such that

1. $\sigma_{j} \in \Delta\left(S_{j}\right)$ is a Borel probability measure over the profiles of signals $s_{j} \equiv\left(s_{j}^{1}, \ldots, s_{j}^{I}\right)$ that principal $j$ sends to the agents, where $S_{j} \equiv S_{j}^{1} \times \ldots \times S_{j}^{I}$ for some collection of Polish spaces $S_{j}^{i}$ of signals from principal $j$ to every agent $i$.

2. $\phi_{j}: S_{j} \times M_{j} \rightarrow \Delta\left(X_{j}\right)$ is a Borel-measurable function assigning a lottery over principal $j$ 's decisions to every profile of signals $s_{j} \in S_{j}$ she sends to the agents and every profile of messages $m_{j} \equiv\left(m_{j}^{1}, \ldots, m_{j}^{I}\right) \in M_{j}$ she receives from them, where $M_{j} \equiv$ $M_{j}^{1} \times \ldots \times M_{j}^{I}$ for some collection of Polish spaces $M_{j}^{i}$ of messages from every agent $i$ to principal $j$.

We assume that $\operatorname{card} \Omega^{i} \leq \operatorname{card} M_{j}^{i}$ for all $i$ and $j$, so that the language through which agent $i$ communicates with principal $j$ is rich enough for him to report his type to her. A (potentially indirect) standard mechanism for principal $j$ is a special case of a mechanism with signals in which $S_{j}^{i}$ is a singleton for all $i$; hereafter, we will often simplify the notation by omitting $\sigma_{j}$ and representing a standard mechanism solely by a Borel-measurable function $\phi_{j}: M_{j} \rightarrow \Delta\left(X_{j}\right)$ describing the principal's response to the messages she receives from the agents. Notice that, for each $s_{j} \in S_{j}, \phi_{j}\left(s_{j}, \cdot\right): M_{j} \rightarrow \Delta\left(X_{j}\right)$ is a standard mechanism. The private signal $s_{j}^{i}$ agent $i$ receives from principal $j$ thus privately informs agent $i$ of how the decision implemented by principal $j$ responds to the messages she receives from the agents; that is, $s_{i}^{j}$ is a private disclosure from principal $j$ to agent $i$ about the mechanism $\phi_{j}\left(s_{j}, \cdot\right)$. The requirement that signal and message spaces be Polish entails no loss of generality; in particular, the universal standard mechanisms of Epstein and Peters (1999) involve uncountable Polish message spaces.

Admissibility A general requirement for defining expected payoffs in the game to be described below is that, for each $j$, the evaluation mapping $\left(\phi_{j}, s_{j}, m_{j}\right) \mapsto \phi_{j}\left(s_{j}, m_{j}\right)$ be

\footnotetext{
${ }^{2}$ The distinction between an allocation and an outcome is relevant when the agents' types are correlated.
} 
measurable. To do so, we must define a measurable structure over the space of admissible functions $\phi_{j}$. If $S_{j}$ and $M_{j}$ are countable, we can take this space to be $\Delta\left(X_{j}\right)^{S_{j} \times M_{j}}$, endowed with the product Borel $\sigma$-field. If $S_{j}$ or $M_{j}$ are uncountable, however, there is no measurable structure over the space of all Borel-measurable functions $\phi_{j}: S_{j} \times M_{j} \rightarrow \Delta\left(X_{j}\right)$ such that the evaluation mapping for principal $j$ is measurable (Aumann (1961)); in that case, there is no choice but to restrict the space of admissible functions $\phi_{j}$. Admissibility can be shown to coincide with the requirement that this space be of bounded Borel class (Aumann (1961), Rao (1971)), which still allows for a rich class of mechanisms for our analysis. We hereafter fix an admissible space $\Phi_{j}$, endowed with a $\sigma$-field $\mathcal{F}_{j}$, so that $\Gamma_{j} \equiv \Delta\left(S_{j}\right) \times \Phi_{j}$ is the space of admissible mechanisms for principal $j$, endowed with the product $\sigma$-field $\mathcal{G}_{j}$ generated by the Borel subsets of $\Delta\left(S_{j}\right)$ and the elements of $\mathcal{F}_{j}$. When attention is restricted to standard mechanisms, the set of admissible mechanisms is simply denoted by $\Phi_{j}$, with the understanding that signal spaces are singletons.

Timing and Strategies The competing-mechanism game $G^{S M}$ with private disclosures unfolds in four stages:

1. the principals simultaneously post mechanisms, observed by all agents;

2. the principals' mechanisms send private signals to the agents;

3. after observing their types, the agents simultaneously send messages to the principals;

4. the principals' decisions are implemented and all payoffs accrue.

A mixed strategy for principal $j$ is a probability measure $\mu_{j} \in \Delta\left(\Gamma_{j}\right)$ over $\mathcal{G}_{j}$. A strategy for agent $i$ is a measurable function $\lambda^{i}: \Gamma \times S^{i} \times \Omega^{i} \rightarrow \Delta\left(M^{i}\right)$ that assigns to every profile of mechanisms $\gamma \equiv\left(\gamma_{1}, \ldots, \gamma_{J}\right) \in \Gamma \equiv \Gamma_{1} \times \ldots \times \Gamma_{J}$ that the principals may post, every profile of signals $s^{i} \equiv\left(s_{1}^{i}, \ldots, s_{J}^{i}\right) \in S^{i} \equiv S_{1}^{i} \times \ldots \times S_{J}^{i}$ that agent $i$ may receive, and every type $\omega^{i} \in \Omega^{i}$ of agent $i$ a Borel probability measure over the profiles of messages $m^{i} \equiv\left(m_{1}^{i}, \ldots, m_{J}^{i}\right) \in M^{i} \equiv M_{1}^{i} \times \ldots \times M_{J}^{i}$ sent by agent $i$, where $\Gamma \times S^{i} \times M^{i}$ is endowed with the appropriate product $\sigma$-field. The allocation $z_{\mu, \lambda}: \Omega \rightarrow \Delta(X)$ induced by the strategies $(\mu, \lambda) \equiv\left(\mu_{1}, \ldots, \mu_{J}, \lambda^{1}, \ldots, \lambda^{I}\right)$ is then defined by

$$
z_{\mu, \lambda}(x \mid \omega) \equiv \int_{\Gamma} \int_{S} \int_{M} \prod_{j=1}^{J} \phi_{j}\left(s_{j}, m_{j}\right)\left(x_{j}\right) \bigotimes_{i=1}^{I} \lambda^{i}\left(\mathrm{~d} m^{i} \mid \gamma, s^{i}, \omega^{i}\right) \bigotimes_{j=1}^{J} \sigma_{j}\left(\mathrm{~d} s_{j}\right) \bigotimes_{j=1}^{J} \mu_{j}\left(\mathrm{~d} \gamma_{j}\right)
$$

for all $(\omega, x) \in \Omega \times X$, where $S \equiv S_{1} \times \ldots \times S_{J}$ and $M \equiv M_{1} \times \ldots \times M_{J}$. For every profile of mechanisms $\gamma$, a behavior strategy for agent $i$ in the subgame $\gamma$ played by the agents is a 
Borel-measurable function $\beta^{i}: S^{i} \times \Omega^{i} \rightarrow \Delta\left(M^{i}\right)$ assigning a Borel probability measure over the profile of messages $m^{i} \in M^{i}$ she sends to the principals to every profile of signals $s^{i} \in S^{i}$ she may receive and to every realization $\omega^{i} \in \Omega^{i}$ of her type. We let $z_{\gamma, \beta}$ be the allocation induced by the profile of behavior strategies $\beta \equiv\left(\beta^{1}, \ldots, \beta^{I}\right)$ in the subgame $\gamma$; the latter is defined in the same way as $z_{\mu, \lambda}$, except that $\gamma$ is fixed and $\lambda^{i}\left(\cdot \mid \gamma, s^{i}, \omega^{i}\right)$ is replaced by $\beta^{i}\left(\cdot \mid s^{i}, \omega^{i}\right)$ for all $i$. We denote by $\lambda^{i}(\gamma)$ the behavior strategy induced by the strategy $\lambda^{i}$ in the subgame $\gamma$.

A degenerate case of the game $G^{S M}$ arises when $S_{j}^{i}$ is a singleton for all $i$ and $j$, so that principals cannot engage into private disclosures and can only post standard mechanisms. To distinguish this situation, we denote by $G^{M}$ the corresponding competing-mechanism game without private disclosures; the games studied by Epstein and Peters (1999) and Yamashita (2010) are prominent examples.

Equilibrium In line with the standard practice of the common-agency literature (Peters (2001), Martimort and Stole (2002)) and the competing-mechanism literature (Epstein and Peters (1999), Yamashita (2010), Peters (2014) and Szentes (2015)), we will assume that the agents treat the mechanisms posted by the principals as given. This means that we can identify any subgame $\gamma \equiv\left(\gamma_{1}, \ldots, \gamma_{J}\right) \in \Gamma$ of $G^{S M}$ with a Bayesian game played by the agents, with type space $S^{i} \times \Omega^{i}$ and action space $M^{i}$ for every agent $i$, and in which the agents' beliefs are pinned down by the prior $\mathbf{P}$ and the signal distributions $\left(\sigma_{1}, \ldots, \sigma_{J}\right)$ to which the principals are committed through the mechanisms they post in $\gamma$, whether or not $\gamma$ is reached on the equilibrium path. The strategy profile $(\mu, \lambda)$ is a perfect Bayesian equilibrium $(\mathrm{PBE})$ of $G^{S M}$ whenever

1. for each $\gamma \in \Gamma,\left(\lambda^{1}(\gamma), \ldots, \lambda^{I}(\gamma)\right)$ is a Bayes-Nash equilibrium (BNE) of the subgame $\gamma$ played by the agents;

2. given the continuation equilibrium strategies $\lambda, \mu$ is a Nash equilibrium of the game played by the principals.

An allocation $z$ is incentive-compatible if, for all $i$ and $\omega^{i} \in \Omega^{i}$,

$$
\omega^{i} \in \underset{\hat{\omega}^{i} \in \Omega^{i}}{\arg \max } \sum_{\omega^{-i} \in \Omega^{-i}} \sum_{x \in X} \mathbf{P}\left[\omega^{-i} \mid \omega^{i}\right] z\left(x \mid \hat{\omega}^{i}, \omega^{-i}\right) u^{i}\left(x, \omega^{i}, \omega^{-i}\right) .
$$

It follows from the definition of a BNE in any subgame played by the agents that any allocation $z_{\mu, \lambda}$ supported by a $\operatorname{PBE}(\mu, \lambda)$ of $G^{S M}$ is incentive-compatible; otherwise, some type $\omega^{i}$ of some agent $i$ would be strictly better off mimicking the strategy $\lambda^{i}\left(\cdot \mid \cdot, \cdot, \hat{\omega}^{i}\right)$ 
of some other type $\hat{\omega}^{i}$ - this is an instance of the revelation principle (Myerson (1982)). This observation implies that, when there is a single principal, any allocation that can be implemented by a mechanism with signals can also be implemented via a direct revelation mechanism; as agents take no payoff-relevant actions, such direct revelation mechanisms involve no private disclosures from the principal to the agents. As we show below, the situation is markedly different when several principals contract with several agents.

\section{A Challenge to Folk Theorems}

In this section, we address the question of whether equilibrium outcomes and payoffs of competing-mechanism games without private disclosures, in which principals are restricted to posting standard mechanisms, yet with potentially rich message spaces, are robust to the possibility for the principals to post mechanisms with signals. This question is especially relevant in light of the fact that, as shown by Yamashita (2010), such games typically lend themselves to folk-theorem-types of results. Notice, for future reference, that similar results are also pervasive in the literature on contractible contracts and reciprocal contracting; see, for instance, Kalai, Kalai, Lehrer, and Samet (2010), Peters and Szentes (2012), Peters (2015), and Szentes (2015).

The construction of Yamashita (2010), which we exploit in Section 3.1 below, is based on the idea that, given rich enough message spaces, each principal's equilibrium mechanism can be made sufficiently flexible to punish other principals' potential deviations. This can be achieved by enabling the agents to recommend to every principal $j$ a (deterministic) direct mechanism $d_{j}: \Omega \rightarrow X_{j}$ selecting a decision for any profile of reported types she may receive from them. Specifically, consider a competing-mechanism game without private disclosures in which every message space $M_{j}^{i}$ is sufficiently rich to enable agent $i$ to recommend any direct mechanism to principal $j$ and to make a report about his type; that is, letting $D_{j}$ be the finite set of all such direct mechanisms, $D_{j} \times \Omega^{i} \subset M_{j}^{i}$ for all $i$ and $j$. Accordingly, a recommendation mechanism $\phi_{j}^{r}$ for principal $j$ stipulates that, if every agent $i$ sends a message $m_{j}^{i} \equiv\left(d_{j}^{i}, \omega^{i}\right) \in D_{j} \times \Omega^{i}$ to principal $j$, then

$$
\phi_{j}^{r}\left(m_{j}^{1}, \ldots, m_{j}^{I}\right) \equiv \begin{cases}d_{j}\left(\omega^{1}, \ldots, \omega^{I}\right) & \text { if } \operatorname{card}\left\{i: d_{j}^{i}=d_{j}\right\} \geq I-1 \\ \bar{x}_{j} & \text { otherwise }\end{cases}
$$

where $\bar{x}_{j}$ is some fixed decision in $X_{j}$; if, instead, some agent $i$ sends a message $m_{j}^{i} \notin D_{j} \times \Omega^{i}$ to principal $j$, then $\phi_{j}^{r}$ treats this message as if it coincided with some fixed element $\left(\bar{d}_{j}, \bar{\omega}_{j}^{i}\right)$ of $D_{j} \times \Omega^{i}$, once again applying rule (1). Intuitively, recommendation mechanisms provide a flexible system of punishments against other principals' potential deviations that can be 
used to support many equilibrium allocations. Indeed, Yamashita (2010) establishes the following folk theorem: if $I \geq 3$, then every deterministic incentive-compatible allocation yielding each principal a payoff at least equal to a well-defined min-max-min payoff bound can be supported in equilibrium. ${ }^{3}$

We now provide an example showing that the possibility for principals to use private disclosures undermines this characterization result. In this example, a folk theorem holds for any competing-mechanism game without private disclosures but with rich enough message spaces; however, a continuum of equilibrium payoff vectors of any such game can no longer be supported when principals can post mechanisms with signals. This shows that equilibrium outcomes and payoffs supported by standard mechanisms need not be robust to private disclosures.

Example 1 Let $J \equiv 2$ and $I \equiv 3$. We denote the principals by $\mathrm{P} 1$ and $\mathrm{P} 2$, and the agents by A1, A2, and A3. The decision sets are $X_{1} \equiv\left\{x_{11}, x_{12}\right\}$ for P1 and $X_{2} \equiv\left\{x_{21}, x_{22}\right\}$ for P2. A1 and A2 can each be of two types, with $\Omega^{1}=\Omega^{2} \equiv\left\{\omega_{L}, \omega_{H}\right\}$, whereas A3 can only be of a single type, which we omit from the notation for the sake of clarity. A1's and A2's types are perfectly correlated: only the states $\left(\omega_{L}, \omega_{L}\right)$ and $\left(\omega_{H}, \omega_{H}\right)$ can occur with positive probability under $\mathbf{P}$.

The players' payoffs are represented in Tables 1 and 2 below, in which the first payoff is that of P2 and the last two payoffs are those of A1 and A2, respectively. P1's and A3's payoffs are constant over $X \times \Omega$ and hence play no role in the analysis.

\begin{tabular}{|c|c|c|}
\hline & $x_{21}$ & $x_{22}$ \\
\hline$x_{11}$ & $5,8,8$ & $5,1,1$ \\
$x_{12}$ & $6,4.5,4.5$ & $6,4.5,4.5$ \\
\hline
\end{tabular}

Table 1: Payoffs in state $\left(\omega_{L}, \omega_{L}\right)$.

\begin{tabular}{|c|c|c|}
\hline & $x_{21}$ & $x_{22}$ \\
\hline$x_{11}$ & $6,4.5,4.5$ & $6,4.5,4.5$ \\
$x_{12}$ & $5,1,1$ & $5,8,8$ \\
\hline
\end{tabular}

Table 2: Payoffs in state $\left(\omega_{H}, \omega_{H}\right)$.

\footnotetext{
${ }^{3}$ As pointed out by Peters (2014), however, these bounds typically depend on the message spaces $M_{j}^{i}$. The requirement that there be at least three agents reflects that, according to (1), near unanimity unequivocally pins down a direct mechanism for each principal posting a recommendation mechanism. Relatedly, Attar, Campioni, Mariotti, and Piaser (2021) show that this and related folk theorems crucially hinge on each agent participating and communicating with each principal, regardless of the profile of posted mechanisms.
} 


\subsection{A Folk Theorem in Standard Mechanisms}

In the context of this example, let us first consider, as in Yamashita (2010), a general competing-mechanism game $G_{1}^{M}$ without private disclosures, and with message spaces such that $D_{j} \times \Omega^{i} \subset M_{j}^{i}$ for all $i$ and $j$, so that principals can post recommendation mechanisms. To guarantee the existence of an equilibrium in every subgame $\phi \equiv\left(\phi_{1}, \phi_{2}\right)$ of $G_{1}^{M}$, we assume that all the message spaces $M_{j}^{i}$ are finite. Our first result characterizes the set of equilibrium payoffs for P2 in $G_{1}^{M}$.

Lemma 1 Any payoff for P2 in [5,6] can be supported in a PBE of $G_{1}^{M}$.

Proof. The proof consists of two steps. Step 1 proves that $G_{1}^{M}$ admits a PBE in which P2 obtains his minimum feasible payoff of 5 , which also shows that 5 is P2's min-max-min payoff in $G_{1}^{M}$. Step 2 then leverages on the construction in Step 1 to prove that any payoff for P2 in $(5,6]$ can also be supported in a PBE of $G_{1}^{M}$, which completes the proof.

Step 1 We first show that the outcome

$$
z\left(\omega_{L}, \omega_{L}\right) \equiv \delta_{\left(x_{11}, x_{21}\right)}, \quad z\left(\omega_{H}, \omega_{H}\right) \equiv \delta_{\left(x_{12}, x_{22}\right)}
$$

in which P2 obtains her minimum feasible payoff of 5 , can be supported in a PBE of $G_{1}^{M}{ }^{4}$ To establish this result, we first show that, if P1 and P2 post recommendation mechanisms, then there exists a continuation BNE supporting the outcome (2). We next show that, in every subgame in which $\mathrm{P} 1$ posts her equilibrium recommendation mechanism, there exists a continuation BNE in which P2 obtains a payoff of 5. The result then follows from these two properties along with the fact that P1 has no profitable deviation as her payoff is constant over $X \times \Omega$.

On Path Suppose that both P1 and P2 post recommendation mechanisms $\phi_{1}^{r}$ and $\phi_{2}^{r}$. We assume that, for each $j, \bar{\omega}_{j}^{1}=\bar{\omega}_{j}^{2}=\omega_{L}$, so that, if some agent $i=1,2$ sends a message $m_{j}^{i} \notin D_{j} \times \Omega^{i}$ to principal $j, \phi_{j}^{r}$ treats this message as if agent $i$ reported to principal $j$ to be of type $\omega_{L}$. We claim that, in the subgame $\left(\phi_{1}^{r}, \phi_{2}^{r}\right)$, it is a BNE for the three agents to recommend the direct mechanisms $\left(d_{1}^{*}, d_{2}^{*}\right)$ defined by

$$
d_{1}^{*}(\omega) \equiv\left\{\begin{array} { l l } 
{ x _ { 1 1 } } & { \text { if } \omega = ( \omega _ { L } , \omega _ { L } ) } \\
{ x _ { 1 2 } } & { \text { otherwise } }
\end{array} \quad \text { and } \quad d _ { 2 } ^ { * } ( \omega ) \equiv \left\{\begin{array}{ll}
x_{21} & \text { if } \omega=\left(\omega_{L}, \omega_{L}\right) \\
x_{22} & \text { otherwise }
\end{array}\right.\right.
$$

for all $\omega \equiv\left(\omega^{1}, \omega^{2}\right) \in \Omega^{1} \times \Omega^{2}$, and for A1 and A2 to report their types truthfully to P1 and P2. To see this, we only need to observe that these strategies implement the outcome

\footnotetext{
${ }^{4}$ For any finite set $A$ and for each $a \in A, \delta_{a}$ is the Dirac measure over $A$ assigning probability 1 to $a$.
} 
(2), which yields A1 and A2 their maximum feasible payoff of 8 in each state; because A3's payoff is constant over $X \times \Omega$, these strategies thus form a BNE of the subgame $\left(\phi_{1}^{r}, \phi_{2}^{r}\right)$. The claim follows.

Off Path Because P1's payoff is constant over $X \times \Omega$, she has no profitable deviation. Suppose then that P2 deviates to some arbitrary standard mechanism $\phi_{2}: M_{2} \rightarrow \Delta\left(X_{2}\right)$, and let $p\left(m_{2}\right)$ be the probability that the lottery $\phi_{2}\left(m_{2}\right)$ assigns to decision $x_{21}$ when the agents send the messages $m_{2} \equiv\left(m_{2}^{1}, m_{2}^{2}, m_{2}^{3}\right) \in M_{2}$ to P2. Now, let

$$
\bar{p} \equiv \max _{m_{2} \in M_{2}} p\left(m_{2}\right)
$$

and select a message profile $\bar{m}_{2} \equiv\left(\bar{m}_{2}^{1}, \bar{m}_{2}^{2}, \bar{m}_{2}^{3}\right) \in M_{2}$ that achieves the maximum in (4); similarly, let

$$
\underline{p} \equiv \min _{\left(m_{2}^{1}, m_{2}^{2}\right) \in M_{2}^{1} \times M_{2}^{2}} p\left(m_{2}^{1}, m_{2}^{2}, \bar{m}_{2}^{3}\right)
$$

and select a message profile $\left(\underline{m}_{2}^{1}, \underline{m}_{2}^{2}\right) \in M_{2}^{1} \times M_{2}^{2}$ for A1 and A2 that, given $\bar{m}_{2}^{3}$, achieves the minimum in (5). That $\bar{p}, \bar{m}_{2}, \underline{p}$, and $\left(\underline{m}_{2}^{1}, \underline{m}_{2}^{2}\right)$ are well-defined for any given $\phi_{2}$ follows from the fact that $M_{2}$ is finite. We now prove that there exist BNE strategies for the agents in the subgame $\left(\phi_{1}^{r}, \phi_{2}\right)$ such that P2 obtains a payoff of 5 , so that the deviation is not profitable. We consider two cases in turn.

Case 1: $\bar{p} \geq \frac{1}{2}$ Suppose first that $\phi_{2}$ is such that $\bar{p} \geq \frac{1}{2}$. We claim that the subgame $\left(\phi_{1}^{r}, \phi_{2}\right)$ admits a BNE that satisfies the following properties: (i) all agents recommend the direct mechanism $d_{1}^{*}$ to $\mathrm{P} 1$, as if $\mathrm{P} 2$ did not deviate; (ii) A1 and A2 truthfully report their types to P1; (iii) A3 sends message $\bar{m}_{2}^{3}$ to P2; (iv) P2 obtains a payoff of 5 . As for (i), the argument is that unilaterally sending a different recommendation to P1 is of no avail as no agent is pivotal. As for (iii), sending $\bar{m}_{2}^{3}$ to P2 is optimal for A3 given that his payoff is constant over $X \times \Omega$. Consider then (ii). Suppose first that the state is $\left(\omega_{L}, \omega_{L}\right)$. Because $\bar{p} \geq \frac{1}{2}, 8 \bar{p}+(1-\bar{p}) \geq 4.5$. From Table 1 , and by definition of $d_{1}^{*}$ and $\bar{m}_{2}$, it thus follows that, if $\mathrm{A} 2$ reports $\omega_{L}$ to $\mathrm{P} 1$ and sends $\bar{m}_{2}^{2}$ to $\mathrm{P} 2$, and if $\mathrm{A} 3$ sends $\bar{m}_{2}^{3}$ to $\mathrm{P} 2$, then A1 best responds by reporting $\omega_{L}$ to $\mathrm{P} 1$ and sending $\bar{m}_{2}^{1}$ to $\mathrm{P} 2$; notice, in particular, that, because $\bar{\omega}_{1}^{1}=\omega_{L}$, if A1 sends a message $m_{1}^{1} \notin D_{1} \times \Omega^{1}$ to $\mathrm{P} 1$, then P1 takes the same decision as if A1 truthfully reported his type to her. The argument for A2 is identical. Suppose next that the state is $\left(\omega_{H}, \omega_{H}\right)$. If either A1 or A2 truthfully reports his type to P1, then, by definition of $d_{1}^{*}$, the other informed agent A2 or A1 cannot induce P1 to take a decision other than $x_{12}$. These properties, along with the finiteness of $M_{2}$, imply that the subgame $\left(\phi_{1}^{r}, \phi_{2}\right)$ admits a BNE 
satisfying (i)-(iii). In this BNE, P1 takes decision $x_{11}$ in state $\left(\omega_{L}, \omega_{L}\right)$ and decision $x_{12}$ in state $\left(\omega_{H}, \omega_{H}\right)$, yielding a payoff of 5 to P2, as required by (iv). The claim follows.

Case 2: $\bar{p}<\frac{1}{2}$ Suppose next that $\phi_{2}$ is such that $\bar{p}<\frac{1}{2}$. We claim that the subgame $\left(\phi_{1}^{r}, \phi_{2}\right)$ admits a BNE that satisfies the following properties: (i) all agents recommend the direct mechanism

$$
d_{1}(\omega) \equiv \begin{cases}x_{12} & \text { if } \omega=\left(\omega_{H}, \omega_{H}\right) \\ x_{11} & \text { otherwise }\end{cases}
$$

to P1; (ii) A1 and A2 truthfully report their types to P1; (iii) A3 sends message $\bar{m}_{2}^{3}$ to P2; (iv) P2 obtains a payoff of 5 . The arguments for (i) and (iii) are the same as in Case 1. Consider then (ii). Suppose first that the state is $\left(\omega_{L}, \omega_{L}\right)$. If either A1 or A2 truthfully reports his type to $\mathrm{P} 1$, then, by definition of $d_{1}$, the other informed agent A2 or A1 cannot induce P1 to take a decision other than $x_{11}$. Suppose next that the state is $\left(\omega_{H}, \omega_{H}\right)$. Because $\underline{p} \leq \bar{p}<\frac{1}{2}, \underline{p}+8(1-\underline{p})>4.5$. From Table 2 , and by definition of $d_{1}$ and $\left(\underline{m}_{2}^{1}, \underline{m}_{2}^{2}\right)$, it thus follows that, if A2 reports $\omega_{H}$ to P1 and sends $\underline{m}_{2}^{2}$ to P2, and if A3 sends $\bar{m}_{2}^{3}$ to P2, then A1 best responds by reporting $\omega_{H}$ to $\mathrm{P} 1$ and sending $\underline{m}_{2}^{1}$ to P2; notice, in particular, that, because $\bar{\omega}_{1}^{1}=\omega_{L}$, if A1 sends a message $m_{1}^{1} \notin D_{1} \times \Omega^{1}$ to P1, then P1 takes the same decision as if A1 misreported his type. The argument for A2 is identical. These properties, along with the finiteness of $M_{2}$, imply that the subgame $\left(\phi_{1}^{r}, \phi_{2}\right)$ admits a BNE satisfying (i)-(iii). The argument for (iv) is then the same as in Case 1. The claim follows.

This completes Step 1 of the proof. We refer to the Appendix for Step 2 of the proof. The result follows.

The arguments in Step 1 of the proof rely on the same intuition as in Yamashita (2010, Theorem 1). The possibility for the agents to recommend a different direct mechanism to P1 for every mechanism posted by P2 allows them to implement punishments contingent on P2's deviations. In particular, the argument in Case 2 shows that any deviation by P2 to a mechanism that implements $x_{21}$ with a probability strictly less than $\frac{1}{2}$ is blocked by recommending to $\mathrm{P} 1$ the direct mechanism $d_{1}$, which is different from the equilibrium mechanism $d_{1}^{*}$. Observe that, unlike in Yamashita (2010), we allow principals to post stochastic mechanisms; yet the threat of agents choosing a deterministic direct mechanism is sufficient to yield P2 her minimum feasible payoff of 5 in equilibrium.

Stochastic mechanisms, however, can be used to support random allocations; see, for instance, Xiong (2013). Step 2 of the proof in turn shows that this permits one to support any payoff for P2 in the feasible set $[5,6]$ in equilibrium. The proof, which is provided in 
the Appendix, only requires adjusting the principals' behavior on path-off path, letting the agents coordinate on the mechanisms $d_{1}^{*}$ or $d_{1}$ used in Step 1 suffices to deter P2's deviations. To this end, we modify Yamashita's (2010) definition of a recommendation mechanism to allow principals to randomize over their decisions on path, while maintaining the assumption that the agents' message spaces are finite.

In related work, Peters and Troncoso-Valverde (2013) establish a folk theorem in a generalized version of Yamashita (2010). In the game they study, any outcome corresponding to an allocation that is incentive-compatible and individually rational in the sense of Myerson (1979) can be supported in equilibrium provided there are at least seven players. It is straightforward to check that the outcome (2) satisfies these conditions, which guarantees that it can also be supported in equilibrium in their framework. ${ }^{5}$ Notice finally that, whereas, in general, a principal's min-max-min payoff may be sensitive to the richness of the available message spaces, in our example P1 posting a recommendation mechanism is sufficient to inflict P2 her minimum feasible payoff of 5 , leaving no role for additional messages beyond those contained in $D_{1} \times \Omega^{i}$ for all $i$. In other words, that P2's relevant min-max-min payoff is equal to 5 is fairly uncontroversial.

\subsection{Nonrobustness to Private Disclosures}

We now show that many of the equilibrium payoffs characterized in Lemma 1 cannot be supported when private disclosures are allowed for. Specifically, Lemma 2 below shows that, in any enlarged game in which principals can post mechanisms with signals, P2 can guarantee herself a payoff strictly higher than her min-max-min payoff of 5 . To this end, we consider a general competing-mechanism game $G_{1}^{S M}$ with private disclosures; this notably includes the case where $D_{j} \times \Omega^{i} \subset M_{j}^{i}$ for all $i$ and $j$, as in the game $G_{1}^{M}$ studied in Section 3.1. To guarantee that the result is not driven by the possible nonexistence of equilibria, we assume that all the signal spaces $S_{j}^{i}$ and the message spaces $M_{j}^{i}$ are finite. ${ }^{6}$

The proof of Lemma 2 crucially exploits the fact that, by posting a mechanism with signals, P2 can asymmetrically inform the agents of her decision. Specifically, we construct a mechanism for P2 such that, when communicating with P1, A1 is perfectly informed of P2's decision, while A2 and A3 are kept in the dark by P2. Such an asymmetry in the information transmitted by P2 to the agents, which is possible only when private disclosures

\footnotetext{
${ }^{5}$ The requirement on the number of players can be met by adding additional agents identical to A3.

${ }^{6}$ As the arguments below reveal, the second part of Lemma 2, which provides a lower bound for P2's payoff in $G_{1}^{S M}$, does not hinge on this simplifying assumption, and extends to any infinite game $G_{1}^{S M}$ that admits an equilibrium.
} 
are allowed for, is precisely what enables P2 to guarantee herself a payoff strictly above her min-max-min payoff of 5 regardless of the mechanism posted by P1 and of the agents' continuation equilibrium strategies.

To see this, notice that the only way to keep P2's payoff down to 5 is for P1 to take decision $x_{11}$ in state $\left(\omega_{L}, \omega_{L}\right)$ and decision $x_{12}$ in state $\left(\omega_{H}, \omega_{H}\right)$. However, by privately informing A1 of her decision, P2 can exploit the fact that, in state $\left(\omega_{L}, \omega_{L}\right)$, and upon learning that $x_{2}=x_{22}$, A1's preferences over $X_{1}$ are perfectly aligned with hers; this guarantees that, if A1 could influence P1's decision in state $\left(\omega_{L}, \omega_{L}\right)$, she would induce P1 to take decision $x_{12}$ with positive probability, bringing P2's payoff strictly above 5 . Hence, given the other agents' messages, A1 must not be able to influence P1's decision in state $\left(\omega_{L}, \omega_{L}\right)$. A similar argument implies that, given the other agents' messages, A1 must not be able to influence P1's decision in state $\left(\omega_{H}, \omega_{H}\right)$ either. ${ }^{7}$ Moreover, because A3 does not observe the state, his message to P1 must be the same in each state. As a result, A2 must de facto have full control over P1's decision. However, when P2 is expected to take decision $x_{21}$ with probability $\sigma>\frac{1}{2}$, A2, without receiving further information from P2, strictly prefers to induce P1 to take decision $x_{11}$ in both state. Hence, if she has the possibility to do so, which we just argued must be the case, she has no incentive to induce the distribution over $X_{1}$ that inflicts the min-max-min payoff of 5 on $\mathrm{P} 2$.

Lemma 2 proves a more general result by characterizing an interval of P2's equilibrium payoffs in $G_{1}^{M}$ that cannot be supported when private disclosures are allowed for.

Lemma $2 G_{1}^{S M}$ admits a PBE. Moreover, if card $S_{2}^{1} \geq 2$, then P2's payoff in any PBE of $G_{1}^{S M}$ is at least equal to $5+\frac{\mathbf{P}\left[\left(\omega_{L}, \omega_{L}\right)\right] \mathbf{P}\left[\left(\omega_{H}, \omega_{H}\right)\right]}{2-\mathbf{P}\left[\left(\omega_{L}, \omega_{L}\right)\right]}$.

Proof. We first show that a PBE exists. We next establish the desired bound on P2's equilibrium payoff.

Existence of a PBE Because, for each $j$, the sets $S_{j}$ and $M_{j}$ are finite, the space $\Gamma_{j} \equiv \Delta\left(S_{j}\right) \times \Delta\left(X_{j}\right)^{S_{j} \times M_{j}}$ of mechanisms for principal $j$ in $G_{1}^{S M}$ is compact, and every subgame $\left(\gamma_{1}, \gamma_{2}\right) \in \Gamma_{1} \times \Gamma_{2}$ is finite; moreover, the agents' information structures and payoffs are continuous functions of $\left(\gamma_{1}, \gamma_{2}\right)$. Hence the BNE of the subgame $\left(\gamma_{1}, \gamma_{2}\right)$ form a nonempty compact set $B^{*}\left(\gamma_{1}, \gamma_{2}\right)$, and the correspondence $B^{*}: \Gamma_{1} \times \Gamma_{2} \rightarrow \prod_{i=1}^{3} \Delta\left(M^{i}\right)^{S^{i} \times \Omega^{i}}$ is upper hemicontinuous (Milgrom and Weber (1985, Theorem 2)) and, therefore, admits a Borel-measurable selection $b^{*} \equiv\left(b^{1 *}, b^{2 *}, b^{3 *}\right)$ by Kuratowski and Ryll-Nardzewski's selection

\footnotetext{
${ }^{7}$ Otherwise, in state $\left(\omega_{H}, \omega_{H}\right)$, and upon learning that $x_{2}=x_{21}$, A1 would induce P1 to take decision $x_{11}$ with positive probability, bringing P2's payoff again strictly above 5 .
} 
theorem (Aliprantis and Border (2006, Theorem 18.13)); the corresponding strategy for every agent $i$ in $G_{1}^{S M}$ is defined by $\lambda^{i *}\left(m^{i} \mid \gamma_{1}, \gamma_{2}, s^{i}, \omega^{i}\right) \equiv b^{i *}\left(\gamma_{1}, \gamma_{2}\right)\left(m^{i} \mid s^{i}, \omega^{i}\right)$. Now, suppose that P1 posts the mechanism $\gamma_{1}^{*}$ that equiprobably randomizes between decisions $x_{11}$ and $x_{12}$ regardless of the signals $\mathrm{P} 1$ sends to the agents and the messages she receives from them. Then, from Tables 1-2, P2 obtains an expected payoff of 5.5 regardless of the mechanism she posts. Because P1's payoff is constant over $X \times \Omega$, it follows that, for each $\gamma_{2}^{*} \in \Gamma_{2}$, $\left(\gamma_{1}^{*}, \gamma_{2}^{*}, \lambda^{1 *}, \lambda^{2 *}\right)$ is a PBE of $G_{1}^{S M}$.

A Tighter Payoff Bound for P2 For each $\sigma \in\left(\frac{1}{2}, 1\right)$, we first construct a mechanism $\gamma_{2}(\sigma) \in \Gamma_{2}$ that guarantees P2 a payoff of $5+\frac{(1-\sigma) \mathbf{P}\left[\left(\omega_{L}, \omega_{L}\right)\right] \mathbf{P}\left[\left(\omega_{H}, \omega_{H}\right)\right]}{1-\sigma \mathbf{P}\left[\left(\omega_{L}, \omega_{L}\right)\right]}$ regardless of the mechanism posted by P1 and of the agents' continuation equilibrium strategies; that is,

$$
\begin{aligned}
& \inf _{\gamma_{1} \in \Gamma_{1}} \inf _{\beta \in B^{*}\left(\gamma_{1}, \gamma_{2}(\sigma)\right)} \sum_{\omega \in \Omega} \sum_{x \in X} \mathbf{P}[\omega] z_{\gamma_{1}, \gamma_{2}(\sigma), \beta}(x \mid \omega) v_{2}(x, \omega) \\
& \geq 5+\frac{(1-\sigma) \mathbf{P}\left[\left(\omega_{L}, \omega_{L}\right)\right] \mathbf{P}\left[\left(\omega_{H}, \omega_{H}\right)\right]}{1-\sigma \mathbf{P}\left[\left(\omega_{L}, \omega_{L}\right)\right]},
\end{aligned}
$$

where $z_{\gamma_{1}, \gamma_{2}(\sigma), \beta}(x \mid \omega)$ is the probability that the decision profile $x$ is implemented when the agents' private information is $\omega$, the principals' mechanisms are $\left(\gamma_{1}, \gamma_{2}(\sigma)\right)$, and the agents play according to $\beta$. To see this, suppose without loss of generality that $\{1,2\} \subset S_{2}^{1}$ and $\emptyset \in S_{2}^{i}$ for $i=2,3$. Fix then some $\sigma \in\left(\frac{1}{2}, 1\right)$, and let $\gamma_{2}(\sigma)$ be the mechanism with signals for P2 such that

- with probability $\sigma_{2}(1, \emptyset, \emptyset) \equiv \sigma$, P2 sends signal $s_{2}^{1}=1$ to A1 and signals $s_{2}^{2}=s_{2}^{3}=\emptyset$ to A2 and A3 and takes decision $x_{21}$ regardless of the profile of messages she receives from the agents;

- with probability $\sigma_{2}(2, \emptyset, \emptyset) \equiv 1-\sigma$, P2 sends signal $s_{2}^{1}=2$ to A1 and signals $s_{2}^{2}=s_{2}^{3}$ $=\emptyset$ to $\mathrm{A} 2$ and $\mathrm{A} 3$ and takes decision $x_{22}$ regardless of the profile of messages she receives from the agents.

Thus, given the private signals sent by P2, A1 knows exactly P2's decision, while A2 and A3 remain uninformed. That is, A2 and A3 believe that P2 takes decision $x_{21}$ with probability $\sigma$ and decision $x_{22}$ with probability $1-\sigma$; yet they know that A1 knows P2's decision. We claim that $\gamma_{2}(\sigma)$ satisfies $(7)$.

Indeed, suppose, by way of contradiction, that there exists $\left(\gamma_{1}, \beta\right) \in \Gamma_{1} \times B^{*}\left(\gamma_{1}, \gamma_{2}(\sigma)\right)$ such that, given $\left(\gamma_{1}, \gamma_{2}(\sigma), \beta\right)$, P2's payoff is $5+\varepsilon$, where

$$
0 \leq \varepsilon<\frac{(1-\sigma) \mathbf{P}\left[\left(\omega_{L}, \omega_{L}\right)\right] \mathbf{P}\left[\left(\omega_{H}, \omega_{H}\right)\right]}{1-\sigma \mathbf{P}\left[\left(\omega_{L}, \omega_{L}\right)\right]}
$$


Observe that the mechanism $\gamma_{2}(\sigma)$ implements decisions in $X_{2}$ that are independent of any messages P2 may receive from the agents and, hence, of any signals sent by $\gamma_{1}$. Thus the only role that signals in $\gamma_{1}$ could play, given $\gamma_{2}(\sigma)$, would be to affect the distribution over P1's decisions induced by the agents; but it follows from standard arguments (Myerson (1982)) that messages are enough to this end, and thus that signals are redundant. We can thus assume that $\gamma_{1}$ is a standard mechanism $\phi_{1}$, involving no signals.

We first establish some useful accounting inequalities. Given $\left(\phi_{1}, \gamma_{2}(\sigma)\right)$ and $\beta$, the probability that $\mathrm{P} 1$ takes decision $x_{11}$ in state $\left(\omega_{L}, \omega_{L}\right)$ can be written as

$$
\pi_{11}\left(\omega_{L}, \omega_{L}\right) \equiv \sigma \pi_{11}\left(\omega_{L}, \omega_{L}, 1\right)+(1-\sigma) \pi_{11}\left(\omega_{L}, \omega_{L}, 2\right)
$$

where, for each $s_{2}^{1} \in\{1,2\}$,

$$
\pi_{11}\left(\omega_{L}, \omega_{L}, s_{2}^{1}\right) \equiv \sum_{\left(m_{1}^{1}, m_{1}^{2}, m_{1}^{3}\right) \in M_{1}} \beta^{1}\left(m_{1}^{1} \mid s_{2}^{1}, \omega_{L}\right) \beta^{2}\left(m_{1}^{2} \mid \omega_{L}\right) \beta^{3}\left(m_{1}^{3}\right) \phi_{1}\left(x_{11} \mid m_{1}^{1}, m_{1}^{2}, m_{1}^{3}\right)
$$

is the probability that P1 takes decision $x_{11}$ in state $\left(\omega_{L}, \omega_{L}\right)$, conditional on P2 sending signal $s_{2}^{1}$ to A1. Similarly, the probability that P1 takes decision $x_{12}$ in state $\left(\omega_{H}, \omega_{H}\right)$ can be written as

$$
\pi_{12}\left(\omega_{H}, \omega_{H}\right) \equiv \sigma \pi_{12}\left(\omega_{H}, \omega_{H}, 1\right)+(1-\sigma) \pi_{12}\left(\omega_{H}, \omega_{H}, 2\right)
$$

where, for each $s_{2}^{1} \in\{1,2\}$,

$$
\pi_{12}\left(\omega_{H}, \omega_{H}, s_{2}^{1}\right) \equiv \sum_{\left(m_{1}^{1}, m_{1}^{2}, m_{1}^{3}\right) \in M_{1}} \beta^{1}\left(m_{1}^{1} \mid s_{2}^{1}, \omega_{H}\right) \beta^{2}\left(m_{1}^{2} \mid \omega_{H}\right) \beta^{3}\left(m_{1}^{3}\right) \phi_{1}\left(x_{12} \mid m_{1}^{1}, m_{1}^{2}, m_{1}^{3}\right)
$$

is the probability that P1 takes decision $x_{12}$ in state $\left(\omega_{H}, \omega_{H}\right)$, conditional on P2 sending signal $s_{2}^{1}$ to A1. By definition of $\varepsilon$, we have

$$
\mathbf{P}\left[\left(\omega_{L}, \omega_{L}\right)\right]\left[6-\pi_{11}\left(\omega_{L}, \omega_{L}\right)\right]+\mathbf{P}\left[\left(\omega_{H}, \omega_{H}\right)\right]\left[6-\pi_{12}\left(\omega_{H}, \omega_{H}\right)\right]=5+\varepsilon,
$$

or, equivalently,

$$
\mathbf{P}\left[\left(\omega_{L}, \omega_{L}\right)\right] \pi_{11}\left(\omega_{L}, \omega_{L}\right)+\mathbf{P}\left[\left(\omega_{H}, \omega_{H}\right)\right] \pi_{12}\left(\omega_{H}, \omega_{H}\right)=1-\varepsilon
$$

which implies

$$
\pi_{11}\left(\omega_{L}, \omega_{L}\right) \geq 1-\frac{\varepsilon}{\mathbf{P}\left[\left(\omega_{L}, \omega_{L}\right)\right]} \quad \text { and } \quad \pi_{12}\left(\omega_{H}, \omega_{H}\right) \geq 1-\frac{\varepsilon}{\mathbf{P}\left[\left(\omega_{H}, \omega_{H}\right)\right]}
$$

as both $\pi_{11}\left(\omega_{L}, \omega_{L}\right)$ and $\pi_{12}\left(\omega_{H}, \omega_{H}\right)$ are at most equal to 1 . Notice that (8) ensures that the right-hand side of each inequality in (13) is strictly positive, and thus can be interpreted 
as a probability as it is at most equal to 1. Similarly, it follows from (9) and from the first inequality in (13) that

$$
\pi_{11}\left(\omega_{L}, \omega_{L}, 2\right) \geq 1-\frac{\varepsilon}{(1-\sigma) \mathbf{P}\left[\left(\omega_{L}, \omega_{L}\right)\right]}
$$

Again, (8) ensures that the right-hand side of (14) is strictly positive, and thus can be interpreted as a probability as it is at most equal to 1 .

We now come to the bulk of the argument. From Table 1 , in state $\left(\omega_{L}, \omega_{L}\right)$, and upon receiving signal $s_{2}^{1}=2$ from P2, A1 wants to minimize the probability that P1 takes decision $x_{11}$. It follows that, given the reporting strategies $\beta^{2}\left(\cdot \mid \omega_{L}\right)$ and $\beta^{3}$ of A2 and A3, any message that A1 sends with positive probability to $\mathrm{P} 1$ in state $\left(\omega_{L}, \omega_{L}\right)$ upon receiving signal $s_{2}^{1}=2$ from P2 induces P1 to take decision $x_{11}$ with probability $\pi_{11}\left(\omega_{L}, \omega_{L}, 2\right)$, and, by (10) and (14), that, for any message $m_{1}^{1} \in M_{1}^{1}$,

$$
\sum_{\left(m_{1}^{2}, m_{1}^{3}\right) \in M_{1}^{2} \times M_{1}^{3}} \beta^{2}\left(m_{1}^{2} \mid \omega_{L}\right) \beta^{3}\left(m_{1}^{3}\right) \phi_{1}\left(x_{11} \mid m_{1}^{1}, m_{1}^{2}, m_{1}^{3}\right) \geq 1-\frac{\varepsilon}{(1-\sigma) \mathbf{P}\left[\left(\omega_{L}, \omega_{L}\right)\right]}
$$

otherwise, by (14), A1 could induce P1 to take decision $x_{11}$ with a probability strictly lower than $\pi_{11}\left(\omega_{L}, \omega_{L}, 2\right)$, yielding A1 a strictly higher payoff, a contradiction. Integrating (15) with respect to the measure $\sigma \beta^{1}\left(\cdot \mid 1, \omega_{H}\right)+(1-\sigma) \beta^{1}\left(\cdot \mid 2, \omega_{H}\right)$ then yields

$$
\begin{gathered}
\sum_{\left(m_{1}^{1}, m_{1}^{2}, m_{1}^{3}\right) \in M_{1}}\left[\sigma \beta^{1}\left(m_{1}^{1} \mid 1, \omega_{H}\right)+(1-\sigma) \beta^{1}\left(m_{1}^{1} \mid 2, \omega_{H}\right)\right] \beta^{2}\left(m_{1}^{2} \mid \omega_{L}\right) \beta^{3}\left(m_{1}^{3}\right) \phi_{1}\left(x_{11} \mid m_{1}^{1}, m_{1}^{2}, m_{1}^{3}\right) \\
\geq 1-\frac{\varepsilon}{(1-\sigma) \mathbf{P}\left[\left(\omega_{L}, \omega_{L}\right)\right]} .
\end{gathered}
$$

This means that, by deviating to $\beta^{2}\left(\cdot \mid \omega_{L}\right)$ in state $\left(\omega_{H}, \omega_{H}\right)$, A2 can ensure that P1 takes decision $x_{11}$ with probability at least $1-\frac{\varepsilon}{(1-\sigma) \mathbf{P}\left[\left(\omega_{L}, \omega_{L}\right)\right]}$. Because $4.5>\sigma+8(1-\sigma)$ as $\sigma>\frac{1}{2}$, A2 can thus guarantee himself a payoff at least equal to

$$
4.5\left\{1-\frac{\varepsilon}{(1-\sigma) \mathbf{P}\left[\left(\omega_{L}, \omega_{L}\right)\right]}\right\}+[\sigma+8(1-\sigma)] \frac{\varepsilon}{(1-\sigma) \mathbf{P}\left[\left(\omega_{L}, \omega_{L}\right)\right]} .
$$

By contrast, if A2 plays $\beta^{2}\left(\cdot \mid \omega_{H}\right)$ in state $\left(\omega_{H}, \omega_{H}\right)$, as he must do in equilibrium, then, by the second inequality in (13), he obtains an expected payoff at most equal to

$$
4.5 \frac{\varepsilon}{\mathbf{P}\left[\left(\omega_{H}, \omega_{H}\right)\right]}+[\sigma+8(1-\sigma)]\left\{1-\frac{\varepsilon}{\mathbf{P}\left[\left(\omega_{H}, \omega_{H}\right)\right]}\right\} .
$$

Comparing (16) and (17), and using again the fact that $4.5>\sigma+8(1-\sigma)$, we obtain that this deviation is profitable for A2 for every $\varepsilon$ satisfying (8), contradicting the assumption that $\beta \in B^{*}\left(\phi_{1}, \gamma_{2}(\sigma)\right)$. Thus $\gamma_{2}(\sigma)$ satisfies $(7)$, as claimed. 
To conclude the proof, observe that, because P2 can, for any $\sigma \in\left(\frac{1}{2}, 1\right)$, guarantee herself a payoff of $5+\frac{(1-\sigma) \mathbf{P}\left[\left(\omega_{L}, \omega_{L}\right)\right] \mathbf{P}\left[\left(\omega_{H}, \omega_{H}\right)\right]}{1-\sigma \mathbf{P}\left[\left(\omega_{L}, \omega_{L}\right)\right]}$ by posting the mechanism $\gamma_{2}(\sigma)$, her payoff in any PBE of $G_{1}^{S M}$ must at least be equal to

$$
\sup _{\sigma \in\left(\frac{1}{2}, 1\right)} 5+\frac{(1-\sigma) \mathbf{P}\left[\left(\omega_{L}, \omega_{L}\right)\right] \mathbf{P}\left[\left(\omega_{H}, \omega_{H}\right)\right]}{1-\sigma \mathbf{P}\left[\left(\omega_{L}, \omega_{L}\right)\right]}=5+\frac{\mathbf{P}\left[\left(\omega_{L}, \omega_{L}\right)\right] \mathbf{P}\left[\left(\omega_{H}, \omega_{H}\right)\right]}{2-\mathbf{P}\left[\left(\omega_{L}, \omega_{L}\right)\right]} .
$$

The result follows.

Lemma 2 constructs a lower bound for P2's equilibrium payoff that is strictly higher than her min-max-min payoff. This lower bound is independent of the richness of the signal spaces $S_{1}^{i}$ and of the message spaces $M_{1}^{i}$ used by $\mathrm{P} 1$ in $G_{1}^{S M}$. In particular, replacing all sums by the appropriate integrals in the proof of Lemma 2 reveals that this bound remains relevant even if some agent can send infinitely many messages to P1-provided, of course, an equilibrium still exists.

Because A1's and A2's preferences are perfectly aligned and A3's payoff is constant over $X \times \Omega$, the reader may wonder why $\mathrm{P} 2$ would want to inform the agents in an asymmetric way. The reason is that, if the agents had the same information about P2's decision, then they could discipline each other, which would enable them to implement incentive-compatible punishments for P2 as in Yamashita's (2010) construction. For example, if all the agents are perfectly informed of P2's decision, then there exists a mechanism for P1 and a continuation equilibrium in the subgame played by the agents that jointly implement the distribution over $X_{1} \times \Omega$ that inflict 5 on P2. The possibility for P2 to asymmetrically inform the agents of her decision is precisely what allows her to prevent the agents from selecting a direct mechanism that punishes her in case she deviates.

Lemmas 1-2 together imply the following result.

Proposition 1 PBE outcomes of competing-mechanism games without private disclosures need not be robust to the possibility for principals to post mechanisms with private signals. In particular, PBE payoff vectors of competing-mechanism games without private disclosures but with rich message spaces such that $D_{j} \times \Omega^{i} \subset M_{j}^{i}$ for all $i$ and $j$ need not be supportable once principals can engage into private disclosures.

\section{A Challenge to Universal Standard Mechanisms}

In the previous section, we have shown that equilibrium outcomes of competing-mechanism games in which principals are restricted to standard mechanisms, yet with potentially rich 
message spaces, need not be robust to the possibility for principals to post mechanisms with signals. In this section, we address the dual question of whether competing-mechanism games in which principals can engage into private disclosures may admit equilibria whose outcomes and payoffs cannot be supported when principals are restricted to standard mechanisms, no matter how rich the message spaces are.

We provide an example showing that this is indeed the case. In this example, a principal can use private signals to make the agents' messages to the other principal depend on information that correlates with her own decision. In turn, this allows the principals to correlate their decisions with the agents' exogenous private information in a way that cannot be achieved, when private disclosures are not feasible, by letting the principals randomize over their choices of mechanisms or by letting the agents randomize over the messages they send to the principals.

Example 2 Let $I=J \equiv 2$. We denote the principals by $\mathrm{P} 1$ and $\mathrm{P} 2$, and the agents by $\mathrm{A} 1$ and $\mathrm{A} 2$. The decision sets are $X_{1} \equiv\left\{x_{11}, x_{12}, x_{13}, x_{14}\right\}$ for P1 and $X_{2} \equiv\left\{x_{21}, x_{22}\right\}$ for P2. A2 can be of two types, with $\Omega^{2} \equiv\left\{\omega_{L}, \omega_{H}\right\}$, whereas A1 can only be of a single type, which we omit from the notation for the sake of clarity. The states $\omega_{L}$ and $\omega_{H}$ are commonly believed to occur with probabilities $\mathbf{P}\left[\omega_{L}\right]=\frac{1}{4}$ and $\mathbf{P}\left[\omega_{H}\right]=\frac{3}{4}$, respectively.

The players' payoffs are represented in Tables 3 and 4 below, in which the first payoff is that of $\mathrm{P} 2$ and the last two payoffs are those of $\mathrm{A} 1$ and $\mathrm{A} 2$, respectively; $\zeta<0$ is an arbitrary loss for P2. P1's payoff is constant over $X \times \Omega$ and hence plays no role in the analysis.

\begin{tabular}{|c|c|c|}
\hline & $x_{21}$ & $x_{22}$ \\
\hline$x_{11}$ & $\zeta, 4,1$ & $\zeta, 8,3.5$ \\
$x_{12}$ & $\zeta, 2,5$ & $\zeta, 9,8$ \\
$x_{13}$ & $10,3,3$ & $\zeta, 5.5,3.5$ \\
$x_{14}$ & $\zeta, 1,3.5$ & $10,7.5,7.5$ \\
\hline
\end{tabular}

Table 3: Payoffs in state $\omega_{L}$.

\begin{tabular}{|c|c|c|}
\hline & $x_{21}$ & $x_{22}$ \\
\hline$x_{11}$ & $\zeta, 1,6$ & $10,7.5,5$ \\
$x_{12}$ & $10,3,9$ & $\zeta, 5.5,6$ \\
$x_{13}$ & $\zeta, 8,7$ & $\zeta, 4.5,7$ \\
$x_{14}$ & $\zeta, 9,6$ & $\zeta, 3,9$ \\
\hline
\end{tabular}

Table 4: Payoffs in state $\omega_{H}$. 


\subsection{An Equilibrium with Private Disclosures}

To illustrate the key ideas in the simplest possible manner, we consider a specific competingmechanism game $G_{2}^{S M}$ with private disclosures in which only P2 can send signals to the agents, and these signals are binary; that is, we let $S_{1}^{1}=S_{1}^{2} \equiv\{\emptyset\}$ and $S_{2}^{1}=S_{2}^{2} \equiv\{1,2\}$. Furthermore, we consider the simplest possible message spaces that allow the agents to report their private information to the principals; that is, we let $M_{1}^{i} \equiv \Omega^{i} \times S_{2}^{i}$ and $M_{2}^{i} \equiv \Omega^{i}$ for all $i .^{8}$ The following result then holds.

Lemma 3 For $\alpha=\frac{2}{3}$, the outcome

$$
\begin{gathered}
z\left(\omega_{L}\right) \equiv \alpha \delta_{\left(x_{13}, x_{21}\right)}+(1-\alpha) \delta_{\left(x_{14}, x_{22}\right)}, \\
z\left(\omega_{H}\right) \equiv \alpha \delta_{\left(x_{12}, x_{21}\right)}+(1-\alpha) \delta_{\left(x_{11}, x_{22}\right)},
\end{gathered}
$$

in which P2 obtains her maximum feasible payoff of 10, can be supported in a PBE of $G_{2}^{S M}$.

Proof. Let P2 post the mechanism $\gamma_{2}^{*} \equiv\left(\sigma_{2}^{*}, \phi_{2}^{*}\right)$ such that

$$
\sigma_{2}^{*}\left(s_{2}\right) \equiv\left\{\begin{array}{cl}
\frac{\alpha}{2} & \text { if } s_{2}=(1,1) \\
\frac{\alpha}{2} & \text { if } s_{2}=(2,2) \\
\frac{1-\alpha}{2} & \text { if } s_{2}=(1,2) \\
\frac{1-\alpha}{2} & \text { if } s_{2}=(2,1)
\end{array}\right.
$$

and, for each $\left(s_{2}, m_{2}\right) \in S_{2} \times M_{2}$,

$$
\phi_{2}^{*}\left(s_{2}, m_{2}\right) \equiv \begin{cases}\delta_{x_{21}} & \text { if } s_{2} \in\{(1,1),(2,2)\} \\ \delta_{x_{22}} & \text { if } s_{2} \in\{(1,2),(2,1)\}\end{cases}
$$

irrespective of the messages $m_{2} \in M_{2}$ received from the agents. A key feature of this mechanism is that, regardless of the signal he receives from P2, every agent's posterior distribution about P2's decision coincides with his prior distribution; that is, each agent believes that P2 takes decision $x_{21}$ with probability $\alpha$ and decision $x_{22}$ with probability $1-\alpha$. For the same reason, each agent believes that the other agent received the same signal as his with probability $\alpha$ and a different signal with probability $1-\alpha$. Thus $\gamma_{2}^{*}$ completely keeps both agents in the dark.

As for P1, let her post the deterministic mechanism $\gamma_{1}^{*} \equiv\left(\delta_{(\emptyset, \emptyset)}, \phi_{1}^{*}\right)$ such that, for each $\left(m_{1}^{1}, m_{1}^{2}\right) \in M_{1}$,

$$
\phi_{1}^{*}\left(\emptyset, \emptyset, m_{1}\right) \equiv \begin{cases}\delta_{x_{13}} & \text { if } m_{1} \in\left\{\left(1, \omega_{L}, 1\right),\left(2, \omega_{L}, 2\right)\right\}, \\ \delta_{x_{14}} & \text { if } m_{1} \in\left\{\left(1, \omega_{L}, 2\right),\left(2, \omega_{L}, 1\right)\right\}, \\ \delta_{x_{12}} & \text { if } m_{1} \in\left\{\left(1, \omega_{H}, 1\right),\left(2, \omega_{H}, 2\right)\right\}, \\ \delta_{x_{11}} & \text { if } m_{1} \in\left\{\left(1, \omega_{H}, 2\right),\left(2, \omega_{H}, 1\right)\right\},\end{cases}
$$

\footnotetext{
${ }^{8}$ As the arguments below reveal, Lemma 3 does not hinge on these simplifying assumptions, and extends to games with richer signal and message spaces as long as $\Omega^{i} \times S_{2}^{i} \subset M_{1}^{i}$ and $\Omega^{i} \subset M_{2}^{i}$ for all $i$.
} 
in which, for instance, $\left(1, \omega_{L}, 1\right)$ stands for $m_{1}^{1}=1$ and $m_{1}^{2}=\left(\omega_{L}, 1\right)$; that is, A1 reports to P1 that she received signal $s_{2}^{1}=1$ from P2, whereas A2 reports that her type is $\omega_{L}$ and that she received signal $s_{2}^{2}=1$ from P2. Observe from (20)-(21) that the outcome (18)-(19) is implemented in the subgame $\left(\gamma_{1}^{*}, \gamma_{2}^{*}\right)$ if every agent reports truthfully to $\mathrm{P} 1$ his type and the signal he receives from $\mathrm{P} 2$. We now show that, if $\alpha=\frac{2}{3}$, then truthful reporting is consistent with a BNE of the subgame $\left(\gamma_{1}^{*}, \gamma_{2}^{*}\right)$. The proof consists of two steps.

Step 1 Consider first A1's incentives, under the belief that A2 is truthful to P1. Because A1 has only one type, we only need to check A1's incentives to truthfully report to P1 the signal he receives from P2.

If A1 truthfully reports his signal to P1, then, regardless of the signal he receives from $\mathrm{P} 2$, his expected payoff is

$$
\begin{aligned}
\frac{1}{4}\left[\alpha u ^ { 1 } \left(x_{13},\right.\right. & \left.\left.x_{21}, \omega_{L}\right)+(1-\alpha) u^{1}\left(x_{14}, x_{22}, \omega_{L}\right)\right] \\
& +\frac{3}{4}\left[\alpha u^{1}\left(x_{12}, x_{21}, \omega_{H}\right)+(1-\alpha) u^{1}\left(x_{11}, x_{22}, \omega_{H}\right)\right]=3 \alpha+7.5(1-\alpha) .
\end{aligned}
$$

If, instead, A1 misreports his signal to P1, then, regardless of the signal he receives from P2, his expected payoff is

$$
\begin{aligned}
& \frac{1}{4}\left[\alpha u^{1}\left(x_{14}, x_{21}, \omega_{L}\right)+(1-\alpha) u^{1}\left(x_{13}, x_{22}, \omega_{L}\right)\right] \\
& \quad+\frac{3}{4}\left[\alpha u^{1}\left(x_{11}, x_{21}, \omega_{H}\right)+(1-\alpha) u^{1}\left(x_{12}, x_{22}, \omega_{H}\right)\right]=\alpha+5.5(1-\alpha),
\end{aligned}
$$

which is strictly less than the value in (22) for all $\alpha \in[0,1]$.

Step 2 Consider next A2's incentives, under the belief that A1 is truthful to P1. We need to check A2's incentives to truthfully report to P1 both his type and the signal he receives from P2.

Case 1: $\omega^{2}=\omega_{L}$ We first consider the behavior of A2 when he is of type $\omega_{L}$. If A2 truthfully reports both his type and his signal to P1, then, regardless of the signal he receives from P2, his expected payoff is

$$
\alpha u^{2}\left(x_{13}, x_{21}, \omega_{L}\right)+(1-\alpha) u^{2}\left(x_{14}, x_{22}, \omega_{L}\right)=3 \alpha+7.5(1-\alpha) .
$$

If, instead, A2 truthfully reports his type but misreports his signal to P1, then, regardless of the signal he receives from $\mathrm{P} 2$, his expected payoff is

$$
\alpha u^{2}\left(x_{14}, x_{21}, \omega_{L}\right)+(1-\alpha) u^{2}\left(x_{13}, x_{22}, \omega_{L}\right)=3.5
$$


which is at most equal to the value in (23) if $\alpha \leq \frac{8}{9}$.

Next, if A2 misreports his type but truthfully reports his signal to P1, then, regardless of the signal he receives from P2, his expected payoff is

$$
\alpha u^{2}\left(x_{12}, x_{21}, \omega_{L}\right)+(1-\alpha) u^{2}\left(x_{11}, x_{22}, \omega_{L}\right)=5 \alpha+3.5(1-\alpha),
$$

which is at most equal to the value in (23) if $\alpha \leq \frac{2}{3}$.

Finally, if A2 misreports both his type and his signal to P1, then, regardless of the signal he receives from $\mathrm{P} 2$, his expected payoff is

$$
\alpha u^{2}\left(x_{11}, x_{21}, \omega_{L}\right)+(1-\alpha) u^{2}\left(x_{12}, x_{22}, \omega_{L}\right)=\alpha+8(1-\alpha),
$$

which is at most equal to the value in (23) if $\alpha \geq \frac{1}{5}$.

Case 2: $\omega^{2}=\omega_{H}$ We next consider the behavior of A2 when he is of type $\omega_{H}$. If A2 truthfully reports both his type and his signal to P1, then, regardless of the signal he receives from $\mathrm{P} 2$, his expected payoff is

$$
\alpha u^{2}\left(x_{12}, x_{21}, \omega_{H}\right)+(1-\alpha) u^{2}\left(x_{11}, x_{22}, \omega_{H}\right)=9 \alpha+5(1-\alpha) .
$$

If, instead, A2 truthfully reports his type but misreports his signal to P1, then, regardless of the signal he receives from $\mathrm{P} 2$, his expected payoff is

$$
\alpha u^{2}\left(x_{11}, x_{21}, \omega_{H}\right)+(1-\alpha) u^{2}\left(x_{12}, x_{22}, \omega_{H}\right)=6
$$

which is at most equal to the value in (24) if $\alpha \geq \frac{1}{4}$.

Next, if A2 misreports his type but truthfully reports his signal to P1, then, regardless of the signal he receives from P2, his expected payoff is

$$
\alpha u^{2}\left(x_{13}, x_{21}, \omega_{H}\right)+(1-\alpha) u^{2}\left(x_{14}, x_{22}, \omega_{H}\right)=7 \alpha+9(1-\alpha)
$$

which is at most equal to the value in (24) if $\alpha \geq \frac{2}{3}$.

Finally, if A2 misreports both his type and his signal to P1, then, regardless of the signal he receives from $\mathrm{P} 2$, his expected payoff is

$$
\alpha u^{2}\left(x_{14}, x_{21}, \omega_{H}\right)+(1-\alpha) u^{2}\left(x_{13}, x_{22}, \omega_{H}\right)=6 \alpha+7(1-\alpha),
$$

which is at most equal to the value in (24) if $\alpha \geq \frac{2}{5}$.

The above analysis implies that it is a BNE for A1 and A2 to truthfully report their private information to $\mathrm{P} 1$ in the subgame $\left(\gamma_{1}^{*}, \gamma_{2}^{*}\right)$ if and only if $\alpha=\frac{2}{3}$. In this continuation 
equilibrium, P2 obtains her maximal feasible payoff of 10. Because P1's payoff is constant over $X \times \Omega$, there exists a PBE of $G_{2}^{S M}$ in which P1 and P2 post the mechanisms $\gamma_{1}^{*}$ and $\gamma_{2}^{*}$, and A1 and A2 play any BNE in any subgame following a deviation by P1 or P2 - the existence of such an equilibrium being guaranteed by the fact that all these subgames are finite. The result follows.

Observe for future reference that, in this equilibrium, A1 obtains an expected payoff of 4.5, while A2 obtains an expected payoff of 4.5 if he is of type $\omega_{L}$ and an expected payoff of $\frac{23}{3}$ if he is of type $\omega_{H}$.

Our equilibrium construction relies on the fact that, although the mechanism with signals $\gamma_{2}^{*}$ is publicly disclosed to both $\mathrm{A} 1$ and $\mathrm{A} 2$, A1 and A2 receive different signals from P2. Specifically, private disclosures by P2 take the form of encryption keys: taken in isolation, each signal sent by P2 is completely uninformative of her decision, whereas, taken together, the two signals sent by P2 are perfectly informative of her decision; we will return to this point in Section 5. Notice in that respect that, if P2 were to inform the agents of her decision, then, after learning that P2 takes decision $x_{21}$, A2, when of type $\omega_{L}$, would no longer be willing to induce P1 to take decision $x_{13}$. By claiming that his type is $\omega_{H}$, type $\omega_{L}$ of A2 can induce P1 to take the decision $x_{12}$ with certainty, obtaining a payoff of 5 instead of the payoff of 3 he obtains by being truthful.

Our construction also reveals that, for P2 to obtain her maximum feasible payoff of 10 while maintaining the agents' incentives, it is essential that both principals randomize over their decisions but do so in a perfectly correlated manner. Whereas it is technically feasible to achieve the equilibrium correlation between the principals' decisions by letting the agents randomize over the messages they send to the principals, while letting the principals respond deterministically to the messages they receive from the agents, such a delegation is not incentive-compatible. It is thus essential that the randomization be carried out by the principals themselves. The correlation between the principals' decisions then requires that some information be passed on from one principal to the other, which, in the absence of a direct communication channel between the principals, is possible only through private disclosures. The analysis in Section 4.2 and the discussion in Section 5 will now confirm this intuition by showing that private signals are indispensable, no matter how rich the message spaces are.

\subsection{Indispensability of Private Signals}

We now show that the outcome (18)-(19) for $\alpha=\frac{2}{3}$ cannot be supported in any equilibrium of 
any game in which the principals are restricted to posting standard mechanisms, irrespective of the richness of the message spaces, and, more generally, that the maximal payoff of 10 for P2 cannot be supported in any equilibrium of any such game. That is, private disclosures are indispensable to support this outcome and this payoff for P2. To this end, we consider a general competing-mechanism game $G_{2}^{M}$ without private disclosures, and with arbitrary message spaces $M_{j}^{i}$. This general formulation notably allows us to capture the case where every principal j's message spaces are large enough — namely, uncountable Polish spaces - to encode the agents' information about the mechanism posted by her opponent, as in Epstein and Peters (1999).

The structure of the argument can be briefly sketched as follows. Suppose, by way of contradiction, that there exists a distribution over pairs of standard mechanisms and a pair of continuation equilibrium strategies for the agents such that P2 obtains her maximum feasible payoff of 10. Then, because the principals' decisions must be perfectly correlated in both states, every pair of mechanisms posted by the principals must respond deterministically to the messages sent by the agents on path. Moreover, because only A2 observes the state, when the distribution over the principals' decisions is state-dependent, A2 must weakly prefer the distribution of messages he is supposed to carry out in each state to the one he is supposed to carry out in the other state. We show that, for every pair of mechanisms posted by the principals, this constrains the joint distribution over the principals' decisions to be the one in (18)-(19) for $\alpha=\frac{2}{3}$; the proof relies on the possibility for A2 to decorrelate the messages he sends to the principals by drawing the message he sends to P1 from his continuation equilibrium strategy in state $\omega_{H}$ and by independently drawing the message he sends to P2 from his continuation equilibrium strategy in state $\omega_{L}$. Another way for A2 to decorrelate his messages to the principals consists in independently drawing twice from his continuation equilibrium strategy in state $\omega_{H}$, and then using the first and the second of these draws to determine his messages to $\mathrm{P} 1$ and $\mathrm{P} 2$, respectively. We show that, for type $\omega_{L}$ of A2 to weakly prefer the distribution over the principals' decisions he is supposed to induce in state $\omega_{L}$ to that induced by this alternative strategy, the messages that A2 sends in state $\omega_{H}$ must have no influence on the principals' decisions when combined with those sent with positive probability by A1. This implies, in turn, that A1 has a profitable deviation, because he can induce the high-payoff decision profile $\left(x_{11}, x_{22}\right)$ in the high-probability state $\omega_{H}$. The following result then holds.

Lemma 4 There exists no PBE of $G_{2}^{M}$ in which P2 obtains her maximum feasible payoff of 10. In particular, there exists no PBE of $G_{2}^{M}$ that supports the outcome (18)-(19) for $\alpha=\frac{2}{3}$. 
Proof. The arguments below more generally show that there is no joint probability measure $\mu \in \Delta\left(\Phi_{1} \times \Phi_{2}\right)$ over $\mathcal{F}_{1} \otimes \mathcal{F}_{2}$ and no equilibrium strategies $\lambda \equiv\left(\lambda^{1}, \lambda^{2}\right)$ for the agents that deliver a payoff of 10 to $\mathrm{P} 2$. In particular, we do not require that $\mu$ be a product measure. In other words, we allow the principals to coordinate their choice of a mechanism through arbitrary correlation devices. The proof is by contradiction, and consists of five steps.

Step 1 Observe first that, with probability 1, $\mu$ must select a pair of mechanisms $\phi \equiv$ $\left(\phi_{1}, \phi_{2}\right)$ such that, in the subgame $\phi$, the equilibrium behavior strategies $\left(\lambda^{1}(\phi), \lambda^{2}(\phi)\right)$ support an outcome of the form

$$
\begin{gathered}
z^{\phi}\left(\omega_{L}\right) \equiv \alpha_{L}^{\phi} \delta_{\left(x_{13}, x_{21}\right)}+\left(1-\alpha_{L}^{\phi}\right) \delta_{\left(x_{14}, x_{22}\right)}, \\
z^{\phi}\left(\omega_{H}\right) \equiv \alpha_{H}^{\phi} \delta_{\left(x_{12}, x_{21}\right)}+\left(1-\alpha_{H}^{\phi}\right) \delta_{\left(x_{11}, x_{22}\right)},
\end{gathered}
$$

for some $\left(\alpha_{L}^{\phi}, \alpha_{H}^{\phi}\right) \in[0,1] \times[0,1]$. Otherwise, with $\mu$-positive probability, P2 would incur a loss $\zeta$, and his overall payoff would be strictly less than 10, a contradiction. The above property implies that, for $\mu$-almost every $\phi$ and for $\left(\lambda^{1}(\phi), \lambda^{2}(\phi)\right)$-almost every message profile $\left(m^{1}, m^{2}\right)$ sent by the agents under the equilibrium behavior strategies $\left(\lambda^{1}(\phi), \lambda^{2}(\phi)\right)$, the lotteries $\left(\phi_{1}\left(m_{1}\right), \phi_{2}\left(m_{2}\right)\right)$ over the principals' decisions must be degenerate.

Step 2 We now prove that, for $\mu$-almost every $\phi, \alpha_{L}^{\phi}=\alpha_{H}^{\phi}=\frac{2}{3}$. Notice first that, as A1 does not know which state prevails, it must be that, given A1's state-independent behavior strategy $\lambda^{1}(\phi)$, the state-dependent outcomes $z^{\phi}\left(\omega_{L}\right)$ and $z^{\phi}\left(\omega_{H}\right)$ are induced by A2's state-dependent behavior strategies $\lambda^{2}(\phi)\left(\cdot \mid \omega_{L}\right)$ and $\lambda^{2}(\phi)\left(\cdot \mid \omega_{H}\right)$. Then, for type $\omega_{L}$ of A2 to induce $z^{\phi}\left(\omega_{L}\right)$ instead of $z^{\phi}\left(\omega_{H}\right)$, it must be that

$$
3 \alpha_{L}^{\phi}+7.5\left(1-\alpha_{L}^{\phi}\right) \geq 5 \alpha_{H}^{\phi}+3.5\left(1-\alpha_{H}^{\phi}\right)
$$

Similarly, for type $\omega_{H}$ of A2 to induce $z^{\phi}\left(\omega_{H}\right)$ instead of $z^{\phi}\left(\omega_{L}\right)$, it must be that

$$
9 \alpha_{H}^{\phi}+5\left(1-\alpha_{H}^{\phi}\right) \geq 7 \alpha_{L}^{\phi}+9\left(1-\alpha_{L}^{\phi}\right)
$$

Summing (25)-(26) yields $\alpha_{L}^{\phi} \leq \alpha_{H}^{\phi}$, and reinserting this inequality in (25)-(26), we obtain

$$
\alpha_{L}^{\phi} \leq \frac{2}{3} \leq \alpha_{H}^{\phi}
$$

Now, consider the alternative behavior strategy for A2 obtained from his state-dependent candidate equilibrium behavior strategies $\lambda^{2}(\phi)\left(\cdot \mid \omega_{L}\right)$ and $\lambda^{2}(\phi)\left(\cdot \mid \omega_{H}\right)$ by decorrelating the two principals' decisions. Formally, this amounts for A2 to independently drawing two message profiles $m^{2} \equiv\left(m_{1}^{2}, m_{2}^{2}\right)$ and $\hat{m}^{2} \equiv\left(\hat{m}_{1}^{2}, \hat{m}_{2}^{2}\right)$ from $\lambda^{2}(\phi)\left(\cdot \mid \omega_{H}\right)$ and $\lambda^{2}(\phi)\left(\cdot \mid \omega_{L}\right)$, 
respectively, and then sending $m_{1}^{2}$ to $\mathrm{P} 1$ and $\hat{m}_{2}^{2}$ to $\mathrm{P} 2$, thus using the distribution $\lambda^{2}(\phi)(\cdot \mid$ $\left.\omega_{H}\right)$ to determine his message to P1 and the distribution $\lambda^{2}(\phi)\left(\cdot \mid \omega_{L}\right)$ to determine his message to P2. Given A1's behavior strategy $\lambda^{1}(\phi)$, this alternative strategy induces a distribution Pr over $\left(x_{11}, x_{12}, x_{21}, x_{22}\right)$ with the following marginals:

$$
\begin{aligned}
& \operatorname{Pr}\left(x_{11}, x_{21}\right)+\operatorname{Pr}\left(x_{11}, x_{22}\right)=1-\alpha_{H}^{\phi}, \\
& \operatorname{Pr}\left(x_{12}, x_{21}\right)+\operatorname{Pr}\left(x_{12}, x_{22}\right)=\alpha_{H}^{\phi}, \\
& \operatorname{Pr}\left(x_{11}, x_{21}\right)+\operatorname{Pr}\left(x_{12}, x_{21}\right)=\alpha_{L}^{\phi}, \\
& \operatorname{Pr}\left(x_{11}, x_{22}\right)+\operatorname{Pr}\left(x_{12}, x_{22}\right)=1-\alpha_{L}^{\phi} .
\end{aligned}
$$

It is easy to check that this system has not full rank, and admits a continuum of solutions indexed by $p \equiv \operatorname{Pr}\left(x_{11}, x_{21}\right)$, which allows us to write $\operatorname{Pr}\left(x_{12}, x_{21}\right)=\alpha_{L}^{\phi}-p, \operatorname{Pr}\left(x_{11}, x_{22}\right)=$ $1-\alpha_{H}^{\phi}-p$, and $\operatorname{Pr}\left(x_{12}, x_{22}\right)=p+\alpha_{H}^{\phi}-\alpha_{L}^{\phi}$. Now, if type $\omega_{L}$ of A2 were to follow the same behavior, thus sending the messages $m_{1}^{2}$ and $\hat{m}_{2}^{2}$ according to the strategy described above, he would obtain an expected payoff of

$$
p+5\left(\alpha_{L}^{\phi}-p\right)+3.5\left(1-\alpha_{H}^{\phi}-p\right)+8\left(p+\alpha_{H}^{\phi}-\alpha_{L}^{\phi}\right)=3.5+0.5 p+4.5 \alpha_{H}^{\phi}-3 \alpha_{L}^{\phi} .
$$

Because this payoff must at most be equal to his equilibrium payoff of $3 \alpha_{L}^{\phi}+7.5\left(1-\alpha_{L}^{\phi}\right)$, it follows that $4 \geq 4.5 \alpha_{H}^{\phi}+1.5 \alpha_{L}^{\phi}$. Combining this inequality with (26), we obtain $\alpha_{L}^{\phi} \geq \alpha_{H}^{\phi}$ and hence $\alpha_{L}^{\phi}=\alpha_{H}^{\phi}=\frac{2}{3}$ by (27), as desired. As a result, in $\mu$-almost every subgame $\phi$, type $\omega_{L}$ of A2 obtains a payoff of 4.5 .

Step 3 Now, fixing a subgame $\phi$ such that $\alpha_{L}^{\phi}=\alpha_{H}^{\phi}=\frac{2}{3}$, consider the alternative behavior strategy for A2 obtained by decorrelating the two principals' decisions, but this time using only the candidate equilibrium behavior strategy $\lambda^{2}(\phi)\left(\cdot \mid \omega_{H}\right)$. Formally, this amounts for A2 to independently drawing two message profiles $m^{2} \equiv\left(m_{1}^{2}, m_{2}^{2}\right)$ and $\hat{m}^{2} \equiv\left(\hat{m}_{1}^{2}, \hat{m}_{2}^{2}\right)$ from $\lambda^{2}(\phi)\left(\cdot \mid \omega_{H}\right)$ and then sending $m_{1}^{2}$ to P1 and $\hat{m}_{2}^{2}$ to P2, thus using the first draw to determine his message to P1 and the second draw to determine his message to P2. Given A1's behavior strategy $\lambda^{1}(\phi)$, this alternative strategy induces a distribution $\tilde{\text { Pr over }}\left(x_{11}, x_{12}, x_{21}, x_{22}\right)$ with the same marginals as under the original strategy,

$$
\begin{aligned}
& \tilde{\operatorname{Pr}}\left(x_{11}, x_{21}\right)+\tilde{\operatorname{Pr}}\left(x_{11}, x_{22}\right)=\frac{1}{3}, \\
& \tilde{\operatorname{Pr}}\left(x_{12}, x_{21}\right)+\tilde{\operatorname{Pr}}\left(x_{12}, x_{22}\right)=\frac{2}{3}, \\
& \tilde{\operatorname{Pr}}\left(x_{11}, x_{21}\right)+\tilde{\operatorname{Pr}}\left(x_{12}, x_{21}\right)=\frac{2}{3}, \\
& \tilde{\operatorname{Pr}}\left(x_{11}, x_{22}\right)+\tilde{\operatorname{Pr}}\left(x_{12}, x_{22}\right)=\frac{1}{3} .
\end{aligned}
$$


It is easy to check that this system too has not full rank, and admits a continuum of solutions indexed by $p \equiv \tilde{\operatorname{Pr}}\left(x_{11}, x_{21}\right)=\tilde{\operatorname{Pr}}\left(x_{12}, x_{22}\right)$, which allows us to write $\tilde{\operatorname{Pr}}\left(x_{11}, x_{22}\right)=\frac{1}{3}-p$ and $\tilde{\operatorname{Pr}}\left(x_{12}, x_{21}\right)=\frac{2}{3}-p$. Now, if type $\omega_{L}$ of A2 were to follow the same behavior, thus sending the messages $m_{1}^{2}$ and $\hat{m}_{2}^{2}$ according to the strategy described above, he would obtain an expected payoff of

$$
p+5\left(\frac{2}{3}-p\right)+3.5\left(\frac{1}{3}-p\right)+8 p=4.5+0.5 p .
$$

Because this payoff must at most be equal to his equilibrium payoff of 4.5 , it follows that $p=0$. This implies that, for $\lambda^{2}(\phi)\left(\cdot \mid \omega_{H}\right) \otimes \lambda^{2}(\phi)\left(\cdot \mid \omega_{H}\right)$-almost every $\left(m^{2}, \hat{m}^{2}\right)$, we have

$$
\left(\phi_{1}\left(m_{1}^{1}, m_{1}^{2}\right), \phi_{2}\left(m_{2}^{1}, \hat{m}_{2}^{2}\right)\right) \in\left\{\delta_{\left(x_{11}, x_{22}\right)}, \delta_{\left(x_{12}, x_{21}\right)}\right\}
$$

for $\lambda^{1}(\phi)$-almost every $m^{1}$. But, according to Step 1 , for $\lambda^{2}(\phi)\left(\cdot \mid \omega_{H}\right) \otimes \lambda^{2}(\phi)\left(\cdot \mid \omega_{H}\right)$-almost every $\left(m^{2}, \hat{m}^{2}\right)$, we have

$$
\begin{aligned}
& \left(\phi_{1}\left(m_{1}^{1}, m_{1}^{2}\right), \phi_{2}\left(m_{2}^{1}, m_{2}^{2}\right)\right) \in\left\{\delta_{\left(x_{11}, x_{22}\right)}, \delta_{\left(x_{12}, x_{21}\right)}\right\}, \\
& \left(\phi_{1}\left(m_{1}^{1}, \hat{m}_{1}^{2}\right), \phi_{2}\left(m_{2}^{1}, \hat{m}_{2}^{2}\right)\right) \in\left\{\delta_{\left(x_{11}, x_{22}\right)}, \delta_{\left(x_{12}, x_{21}\right)}\right\}
\end{aligned}
$$

for $\lambda^{1}(\phi)$-almost every $m_{1}$. Thus $(28)$ implies that for $\lambda^{2}(\phi)\left(\cdot \mid \omega_{H}\right) \otimes \lambda^{2}(\phi)\left(\cdot \mid \omega_{H}\right)$-almost every $\left(m^{2}, \hat{m}^{2}\right)$, we have

$$
\left(\phi_{1}\left(m_{1}^{1}, m_{1}^{2}\right), \phi_{2}\left(m_{2}^{1}, m_{2}^{2}\right)\right)=\left(\phi_{1}\left(m_{1}^{1}, \hat{m}_{1}^{2}\right), \phi_{2}\left(m_{2}^{1}, \hat{m}_{2}^{2}\right)\right)
$$

for $\lambda^{1}(\phi)$-almost every $m_{1}$. Because $\phi_{1}$ and $\phi_{2}$ are measurable, we can then conclude from Fubini's theorem (Aliprantis and Border (2006, Theorem 11.27)) that (29) holds for $\lambda^{1}(\phi) \otimes$ $\lambda^{2}(\phi)\left(\cdot \mid \omega_{H}\right) \otimes \lambda^{2}(\phi)\left(\cdot \mid \omega_{H}\right)$-almost every $\left(m^{1}, m^{2}, \hat{m}^{2}\right)$. Applying again Fubini's theorem, we obtain that for $\lambda^{1}(\phi)$-almost every $m_{1},(29)$ holds for $\lambda^{2}(\phi)\left(\cdot \mid \omega_{H}\right) \otimes \lambda^{2}(\phi)\left(\cdot \mid \omega_{H}\right)$-almost every $\left(m_{2}, \hat{m}_{2}\right)$, so that the mapping $\left(m_{1}^{2}, m_{2}^{2}\right) \mapsto\left(\phi_{1}\left(m_{1}^{1}, m_{1}^{2}\right), \phi_{2}\left(m_{2}^{1}, m_{2}^{2}\right)\right)$ is constant over a set of $\lambda^{2}(\phi)\left(\cdot \mid \omega_{H}\right)$-measure 1 .

Step 4 We are now ready to complete the proof. The upshot from Step 3 is that A1 can force the decision when the state is $\omega_{H}$. This implies that $M^{1}$ should include a message profile allowing A1 to implement $\delta_{\left(x_{11}, x_{22}\right)}$ regardless of the message sent in equilibrium by A2. By sending this message, A1 can achieve a payoff of 7.5 when the state is $\omega_{H}$. Thus, he can guarantee himself an expected payoff of at least $\frac{3}{4} \times 7.5$, which is strictly higher than his equilibrium payoff of 4.5 , a contradiction. The result follows.

It should be noted that the result in Lemma 4 holds no matter how rich the message spaces are. Hence, it also applies to the Epstein and Peters (1999) class of universal mechanisms, 
which, while they allow the agents to communicate all their market information to the principals, nonetheless remain standard mechanisms.

Lemmas 3-4 together imply the following result.

Proposition 2 PBE outcomes and PBE payoff vectors of competing-mechanism games with private disclosures need not be supported in any PBE of any competing-mechanism game without private disclosures-including, in particular, the game in which principals can post universal mechanisms - and this, even if the principals or the agents play mixed strategies in equilibrium..$^{9}$

This result shows that the universal mechanisms of Epstein and Peters (1999) fail to be canonical when principals can engage into private disclosures, that is, when they can send private signals to the agents about their decisions as a way of correlating their decisions with those of the other principals and with the agents' exogenous private information. Together with Proposition 1, Proposition 2 implies that the sets of equilibrium outcomes and payoffs of competing-mechanism games with and without private disclosures are not nested.

Incidentally, Proposition 2 also implies that the folk theorem of Yamashita (2010) does not extend to stochastic allocations. Indeed, the allocation $\left(z\left(\omega_{L}\right), z\left(\omega_{H}\right)\right)$ defined by $(18)-$ (19) for $\alpha=\frac{2}{3}$ is certainly incentive-compatible; moreover, it yields P2 her maximum feasible payoff of 10, which is certainly at least equal to her min-max-min payoff, as defined by Yamashita (2010) over recommendation mechanisms. Yet Lemma 4 implies that this allocation cannot be supported in an equilibrium of $G_{2}^{M}$, even when $D_{j} \times \Omega^{i} \subset M_{j}^{i}$ for all $i$ and $j$, so that recommendation mechanisms are feasible.

Finally, the proof of Lemma 4 does not make use of the property that the principals choose their mechanisms independently. The result in Lemma 4 thus carries over to the case where $G_{2}^{M}$ is augmented by arbitrarily rich public randomizing devices that the principals may use to correlate their choices of mechanisms. On the other hand, the result does hinge on the principals not having access to private randomizing devices whose realizations are not known to the agents at the time they send they messages to the principals; we discuss this issue at length in Section 5.3.

\section{Discussion}

In this section, we put our results in perspective by discussing the different roles that private disclosures play in our examples and by examining the consequence for our results

\footnotetext{
${ }^{9}$ Epstein and Peters (1999) restrict attention to equilibria in which principals play pure strategies.
} 
of alternative informational and contracting assumptions.

\subsection{Informative versus Uninformative Signals}

Each of our examples is designed to illustrate a specific role of private signals; we now discuss these different roles in turn.

In Example 2, private signals are used by P2 on path to correlate her decisions with P1's decisions and the agents' private information in a way that cannot be achieved in equilibrium with standard mechanisms. As we show in the proof of Lemma 3, the private signals that P2 may use to this end need not modify the agents' beliefs. Instead, they can work as pure encryption keys: in isolation, each key is completely uninformative of P2's decision but, taken together, the keys perfectly reveal it.

By contrast, in Example 1, the main thrust of private signals is the destabilizing role they play off path in undermining the robustness of equilibria in standard mechanisms. As we show in the proof of Lemma 2, P2 can guarantee herself a payoff strictly above her minimum feasible payoff by asymmetrically informing the agents about her decision, changing A1's beliefs before A1 has the opportunity to communicate with P1, while keeping A2 and A3 in the dark. In the discussion of Lemma 2, we argued that, if P2 were to perfectly inform all agents of her decision, or, more generally, of the decisions taken in response to the agents' messages - as in a standard mechanism - then it would be possible for P1 to post a mechanism that would inflict on P2 her minimum feasible payoff. We now show that the same conclusion is true of any signal structure that keeps all agents in the dark. Formally, we show that the analog of Lemma 2 is false if P2 is restricted to posting mechanisms in which private signals take the form of uninformative encryption keys as those used in the context of Example 2.

To see this, consider the game $G_{1}^{S M}$ studied in Lemma 2; moreover, as in Lemma 1, assume that $D_{j} \times \Omega^{i} \subset M_{j}^{i}$ for all $i$ and $j$, so that recommendation mechanisms are feasible, and assume that all the message spaces $M_{j}^{i}$ are finite. We say that a mechanism $\gamma_{2} \equiv\left(\sigma_{2}, \phi_{2}\right)$ of $\mathrm{P} 2$ has uninformative signals if

$$
\sum_{s_{2}^{-i} \in S_{2}^{-i}} \sigma_{2}\left(s_{2}^{-i} \mid s_{2}^{i}\right) \phi_{2}\left(x_{2} \mid s_{2}^{i}, s_{2}^{-i}, m_{2}\right)=\sum_{s_{2} \in S_{2}} \sigma_{2}\left(s_{2}\right) \phi_{2}\left(x_{2} \mid s_{2}, m_{2}\right)
$$

for all $i, s_{2}^{i} \in S_{2}^{i}, m_{2} \in M_{2}$, and $x_{2} \in X_{2} \cdot{ }^{10}$ That is, the signals $s_{2}^{i}$ sent by $\mathrm{P} 2$ to any given agent $i$ do not reveal to him anything about P2's effective decision rule $\phi_{2}\left(\cdot \mid s_{2}, \cdot\right)$. The

\footnotetext{
${ }^{10}$ That $S_{2}$ is a finite set plays no role for this discussion. In particular, Lemma 5 below remains valid if $S_{2}$ is an arbitrary Polish space.
} 
following result then holds.

Lemma 5 In $G_{1}^{S M}$, if P1 posts a recommendation mechanism $\phi_{1}^{r}$, then, for every mechanism $\gamma_{2}$ of P2 that has uninformative signals, there exists a BNE of the subgame $\left(\phi_{1}^{r}, \gamma_{2}\right)$ in which P2 obtains her minimum feasible payoff of 5.

Proof. Because A3's payoff is constant over $X \times \Omega$ and A1's and A2's payoff functions are identical, we can focus on A1's incentives. Suppose that, in the subgame $\left(\phi_{1}^{r}, \gamma_{2}\right)$, A2 and A3 play behavior strategies $\beta^{2}$ and $\beta^{3}$ that prescribe the same play for any signals $s_{2}^{2}$ and $s_{2}^{3}$ they may receive from $\mathrm{P} 2$, respectively; that is, for each $\omega^{2} \in \Omega^{2}, \beta^{2}\left(\cdot \mid s_{2}^{2}, \omega^{2}\right)$ is independent of $s_{2}^{2}$, and similarly $\beta^{3}\left(\cdot \mid s_{2}^{3}\right)$ is independent of $s_{2}^{3}$. Then, because every signal A1 receives from P2 is uninformative, A1 may as well best respond by playing a behavior strategy $\beta^{1}$ that prescribes the same play for any signal $s_{2}^{1}$ he may receive from P2; that is, for each $\omega^{1} \in \Omega^{1}, \beta^{1}\left(\cdot \mid s_{2}^{1}\right)$ is independent of $s_{2}^{1}$. Because all the message spaces $M_{j}^{i}$ are finite, this implies that the subgame $\left(\phi_{1}^{r}, \gamma_{2}\right)$ admits a BNE in which all agents play behavior strategies that prescribe the same play for any signals they may receive from P2. According to (30), any such BNE of the subgame $\left(\phi_{1}^{r}, \gamma_{2}\right)$ can be straightforwardly turned into a BNE of the subgame $\left(\phi_{1}^{r}, \hat{\phi}_{2}\right)$ in which P1 posts the recommendation mechanism $\phi_{1}^{r}$ and P2 posts the standard mechanism $\hat{\phi}_{2}$ defined by

$$
\hat{\phi}_{2}\left(x_{2} \mid m_{2}\right) \equiv \sum_{s_{2} \in S_{2}} \sigma_{2}\left(s_{2}\right) \phi_{2}\left(x_{2} \mid s_{2}, m_{2}\right)
$$

for all $m_{2} \in M_{2}$ and $x_{2} \in X_{2}$. Notice that, by construction, the same outcome is implemented in either case. Conversely, any BNE of the subgame $\left(\phi_{1}^{r}, \hat{\phi}_{2}\right)$ can be straightforwardly turned into a BNE of the subgame $\left(\phi_{1}^{r}, \gamma_{2}\right)$ in which all agents play behavior strategies that prescribe the same play for any signals they may receive from $\mathrm{P} 2$, and which implements the same outcome. To conclude, observe that, as $\hat{\phi}_{2}$ is a standard mechanism, we know from the proof of Lemma 1 that the subgame $\left(\phi_{1}^{r}, \hat{\phi}_{2}\right)$ admits a BNE in which P2 obtains a payoff of 5 . The result follows.

This last result reflects that, if P1 posts a recommendation mechanism $\phi_{1}^{r}$ and $\mathrm{P} 2$ posts a mechanism $\gamma_{2}$ with uninformative signals, then there exists a one-to-one correspondence between the babbling equilibria of the subgame $\left(\phi_{1}^{r}, \gamma_{2}\right)$, in which the agents ignore the signals they receive from P2, and the equilibria of the subgame $\left(\phi_{1}^{r}, \hat{\phi}_{2}\right)$ in which P2 posts the standard mechanism $\hat{\phi}_{2}$ obtained by averaging $\gamma_{2}$ over the profiles of signals $s_{2}$. But then, because, by Lemma 1, P2's payoff can be kept down to 5 in the latter case, this must also be 
the true in the former case. Notice that there is no tension between this result and Lemma 3 , which illustrates the power of mechanisms with uninformative signals in the context of Example 2; indeed, the key step in the proof of Lemma 3 precisely consists in constructing a non-babbling equilibrium of the agents' subgame in which they truthfully report to P1 the uninformative signals they receive from P2.

However, Lemma 5 points at a potential drawback of mechanisms with uninformative signals, namely, that they naturally lend themselves to babbling equilibria: if all agents but one ignore their uninformative signals, then the remaining agent may as well do the same. This contrasts with the mechanism constructed in the proof of Lemma 2, which, by disclosing P2's decision asymmetrically to the agents, allows P2 to guarantee herself more than her min-max-min payoff regardless of the mechanism posted by P1 and of the continuation equilibrium played by the agents. The general lesson that Lemma 2 thus illustrates is that, if a principal deviates and makes an informative private disclosure about her decision rule to an agent, then the agent cannot simply ignore it; this reasoning, of course, fully exploits the logic of sequential rationality and the standard assumption that the agents treat the mechanisms posted by the principals as given.

\subsection{Contractible Contracts and Reciprocal Mechanisms}

The nonrobustness result in Proposition 1 is established under the assumption that no principal can directly condition the decisions she takes and/or the mechanism she chooses on the other principals' decisions and/or mechanisms. However, the result extends to settings in which such conditioning is feasible, as in the literature on contractible contracts (Kalai, Kalai, Lehrer, Samet (2010), Peters and Szentes (2012), Szentes (2015)) and reciprocal mechanisms (Peters (2015)). To see this, observe that, in Example 1, the only way to inflict on P2 her minimum feasible payoff of 5 is for P1 to take decision $x_{11}$ in state $\left(\omega_{L}, \omega_{L}\right)$ and decision $x_{12}$ in state $\left(\omega_{H}, \omega_{H}\right)$ with probability 1 . However, because the state is privately observed by A1 and A2, P1 must ultimately let them determine which decisions to implement in response to a deviation by P2, were P2 to post a mechanism with signals. Now suppose, for instance, that, as in the proof of Lemma 2, P2 posts a mechanism whereby she selects a decision at random and only informs $\mathrm{A} 1$ of her decision. Because, when P2 selects decision $x_{22}$, this mechanism perfectly aligns P2's and A1's preferences in each state, P1's mechanism must not be responsive to A1's messages on pain of moving P2's payoff away from 5; notice that this remains true even if P1 can condition the decision she takes and/or the mechanism she chooses on P2's decision and/or mechanism. Thus P1 must entirely delegate to A2 the 
task of making her decision contingent on the state. Yet, by construction, A2 does not know which decision P2 is committed to; moreover, the additional possibility for P1 to, for instance, condition her decision on P2's is of little use if P2's payoff is to be kept down to 5, as this requirement uniquely pins down P1's decision in each state. It follows that P1 still has no way to reward A2 for truthfully reporting the state to her, and, as in the proof of Lemma 2, that A2 has a profitable deviation. We conclude that, even if P1 can resort to contractible contracts or post a reciprocal mechanism, it is impossible for her to induce A1 and A2 to carry out the punishments necessary to block P2's deviation.

In this respect, the case of Proposition 2 is less clear cut. On the one hand, in Example 2, the proof of Lemma 4 goes through unaltered even if each principal can condition her choice of a mechanism on the other principal's mechanism - indeed, the argument is valid for any pair of mechanisms posted by the principals, no matter how this pair is generated. This implies that Proposition 2 extends to settings in which principals can post reciprocal mechanisms (Peters (2015)). On the other hand, Lemma 4 does hinge on each principal being unable to directly condition her decision on the other principal's decision. Indeed, if this were possible, then the outcome (18)-(19) for $\alpha=\frac{2}{3}$ could trivially be supported even without private disclosures.

\subsection{Additional Randomizing Devices}

The constructions in Epstein and Peters (1999) and Yamashita (2010) do not allow for additional randomizing devices that would directly enable principals and agents to correlate their choices. We have closely followed these authors in that respect; indeed, the whole point of Example 2 is to show that such correlation can endogenously arise in equilibrium when principals can post mechanisms with signals, but not when they can only post standard mechanisms. It is nevertheless natural to ask to which extent our findings are robust to the availability of additional randomizing devices.

In Example 1, the destabilizing role of private disclosures remains relevant regardless of whether and at which stage of the game the principals and the agents have access to such devices - they may be used to correlate principals' choices of mechanisms, the messages sent by the agents to the principals, or the decisions taken by the principals in response to these messages. To see this, we can focus on the agents' behavior because, as pointed out in Section 5.1, the key role of private signals in the game $G_{1}^{S M}$ of Example 1 is that they can be used by P2 to eschew equilibrium outcomes supported by standard mechanisms in which she obtains her minimum feasible payoff of 5 . In that respect, enabling the agents 
to observe the realization of a public randomization device - a sunspot - would not change the conclusion of Lemma 2 as the mechanism for P2 constructed in the proof guarantees her a payoff strictly above 5 regardless of the continuation equilibrium played by the agents. Less obviously, this mechanism is still effective regardless of the mechanism posted by P1 even if the agents' behavior in the corresponding subgame is coordinated by a mediator who can first elicit information from the agents and then send them private recommendations. In line with the discussion in Section 5.2, the point is that, once A1 knows that P2 has selected decision $x_{22}$, he has, state-by-state, the same preferences as P2. This means that the mediator cannot extract from A1 information about the state and P2's decision and use that information to keep P2's payoff down to 5. But then the task of punishing P2 must be fully delegated to A2, which we know is impossible.

The situation in Example 2 is slightly different. As mentioned in our discussion of Proposition 2, it is impossible to support the outcome (18)-(19) for $\alpha=\frac{2}{3}$ in the game $G_{2}^{M}$ even if the principals can correlate their choices of standard mechanisms. Moreover, the proof of Lemma 4 goes through unaltered even if agents can coordinate their messages by means of a public randomization device; to see this, we need only notice that, for any pair of mechanisms posted by P1 and P2, and for any realization of the sunspot that enables the agents to correlate their messages, A1 and A2 must play an equilibrium in the continuation game. Thus Proposition 2 extends to settings in which the principals and the agents have access to rich public randomizing devices whose realizations are observed by all players before committing to their choices. Things would be different if the principals could use randomization devices whose realizations are not known to the agents at the time they send their messages to the principals. Indeed, because the only role of private disclosures in the example is to pass on information from one principal to the other without changing the agents' beliefs, such private disclosures can be dispensed with if the principals have access to private correlation devices - that is, to devices whose realization is determined after the agents have sent their messages but before the principals have selected their payoff-relevant decisions. The value of the example is rather to show that, in the setting that has been at the center stage of the literature - in which direct communication between the principals is not feasible, private randomizing devices are not available, and principals cannot condition their decisions on other principals' decisions - private disclosures have important implications for the set of equilibrium outcomes and for the validity of the universal revelation principle established in this setting. 


\section{Concluding Remarks}

Private disclosures, that is, signals about the decisions implemented in a mechanism sent by a principal to the agents before they have an opportunity to send messages back to her, have been ignored in previous work on competing-mechanism games. Our examples show that private disclosures have important consequences for the equilibrium outcomes of such games. They have a bearing on the two pillars of the literature, namely, the canonicity of universal mechanisms (Epstein and Peters (1999)) and the validity of folk theorems (Yamashita (2010)).

Taken together, our two examples illustrate that private signals in competing-mechanism games play a fundamentally different role from the one they play in single-principal settings (Myerson (1982)). In the latter, signals are used to correlate the agents' behavior when the agents take payoff-relevant actions that are not under the control of the principal. In competing-mechanism games, instead, signals are used to asymmetrically inform the agents of the decisions taken by a principal in response to the messages she receives from them. Keeping the agents in the dark allows a principal to eschew punishment from the other principals (Example 1) and to support correlated outcomes and corresponding payoffs that could not be supported otherwise (Example 2).

An open question is what structure for the signal and message space is fully canonical, in the sense of (1) enabling one to support all equilibrium allocations of competing-mechanism games with arbitrarily richer message and signal spaces, and (2) guaranteeing that the equilibrium outcomes of the canonical game are robust to deviations to mechanisms with richer message and signal spaces. Identifying a canonical extensive form, a canonical class of mechanisms, and a set of fully robust equilibrium outcomes for competing-mechanism games are important next steps for future research.

Finally, we have deliberately framed our examples as abstract games, so as to identify and illustrate the different roles that private signals can play in competing-mechanism games. It will be important, in future research, to assess the consequences of private signals in more structured economic environments.

\section{Appendix}

Proof of Lemma 1. We start with a definition. An extended recommendation mechanism $\tilde{\phi}_{j}^{r}: M_{j} \mapsto \Delta\left(X_{j}\right)$ for principal $j$ implements the same decisions as the recommendation mechanism $\phi_{j}^{r}$ in $(1)$, except if at least $I-1$ agents send messages $m_{j}^{i} \equiv\left(d_{j}^{0}, \omega^{i}\right) \in D_{j} \times \Omega^{i}$ 
to principal $j$, for some fixed direct mechanism $d_{j}^{0} \in D_{j}$, in which case principal $j$ disregards $d_{j}^{0}$ and implements a (possibly stochastic) direct mechanism $\tilde{d}_{j}: \Omega \rightarrow \Delta\left(X_{j}\right)$; again, if some agent $i$ sends a message $m_{j}^{i} \notin D_{j} \times \Omega^{i}$ to principal $j$, then $\tilde{\phi}_{j}^{r}$ treats this message as if it coincided with some fixed element $\left(\bar{d}_{j}, \bar{\omega}_{j}^{i}\right)$ of $D_{j} \times \Omega^{i}$, for some $\bar{d}_{j} \neq d_{j}^{0}$.

Step 2 We now construct a family of PBEs of $G_{1}^{M}$, indexed by P2's payoff $v \in(5,6]$, in which P2 posts the same recommendation mechanism $\phi_{2}^{r}$ as in Step 1 of the proof and P1 posts an extended recommendation mechanism $\tilde{\phi}_{1}^{r}$. Again, because P1's payoff is constant over $X \times \Omega$, she has no profitable deviation. If P2 deviates to some arbitrary standard mechanism $\phi_{2}: M_{2} \rightarrow \Delta\left(X_{2}\right)$, then we require that the agents' strategies implement the same punishments for P2 as in Step 1 of the proof. We suppose in particular that the direct mechanism $d_{1}^{0}$ differs from the direct mechanisms $d_{1}^{*}$ and $d_{1}$, defined by (3) and (6), which may be recommended by the agents to P1 following a deviation by P2; recall that these punishments inflict on P2 her minimum feasible payoff of 5. We consider two cases in turn.

Case 1: $v \in(5,5.5]$ We specify $\tilde{\phi}_{1}^{r}$ as follows. First, we assume that $\bar{\omega}_{1}^{1}=\bar{\omega}_{1}^{2}=\omega_{L}$, so that, if some agent $i=1,2$ sends a message $m_{1}^{i} \notin D_{1} \times \Omega^{i}$ to P1, then $\tilde{\phi}_{1}^{r}$ treats this message as if agent $i$ reported to principal $j$ to be of type $\omega_{L}$; recall from Step 1 of the proof that $\phi_{2}^{r}$ similarly satisfies $\bar{\omega}_{2}^{1}=\bar{\omega}_{2}^{2}=\omega_{L}$. Fixing some $\xi \in\left[\frac{1}{2}, 1\right)$, we then let

$$
\tilde{d}_{1}(\omega) \equiv \begin{cases}\tilde{x}_{1}^{\xi} & \text { if } \omega=\left(\omega_{L}, \omega_{L}\right) \\ \tilde{x}_{1}^{1-\xi} & \text { otherwise }\end{cases}
$$

where

$$
\tilde{x}_{1}^{\xi} \equiv \xi \delta_{x_{11}}+(1-\xi) \delta_{x_{12}} \quad \text { and } \quad \tilde{x}_{1}^{1-\xi} \equiv(1-\xi) \delta_{x_{11}}+\xi \delta_{x_{12}}
$$

We now show that, for each $\xi \in\left[\frac{1}{2}, 1\right)$, the subgame $\left(\tilde{\phi}_{1}^{r}, \phi_{2}^{r}\right)$ admits a BNE in which: (i) each agent recommends to $\mathrm{P} 1$ the direct mechanism $d_{1}^{0}$, and recommends to P2 the direct mechanism $d_{2}^{*}$ defined by (3); (ii) A1 and A2 truthfully report their types to P1 and P2. The corresponding payoff for P2 in the subgame $\left(\tilde{\phi}_{1}^{r}, \phi_{2}^{r}\right)$ is $v=6-\xi \in(5,5.5]$ as $\xi$ varies in $\left[\frac{1}{2}, 1\right)$, as desired. Because A3's payoff is constant over $X \times \Omega$, we only need to focus on A1's and A2's incentives.

Consider first state $\left(\omega_{L}, \omega_{L}\right)$, and suppose that A2 and A3 recommend $d_{1}^{0}$ to P1 and $d_{2}^{*}$ to P2, and that A2 truthfully reports his type to P1 and P2. Because A1 is not pivotal, recommending a different direct mechanism to either principal is of no avail to him; moreover, because $\bar{\omega}_{1}^{1}=\bar{\omega}_{2}^{1}=\omega_{L}$, sending a message $m_{j}^{1} \notin D_{j} \times \Omega^{1}$ to any principal $j$ amounts for A1 to truthfully reporting his type to her. We can thus with no loss of generality assume that 
A1 recommends $d_{1}^{0}$ to $\mathrm{P} 1$ and $d_{2}^{*}$ to $\mathrm{P} 2$, and we only need to study A1's reporting decisions. (1) If A1 truthfully reports his type to P1 and P2, then P1 implements the lottery $\tilde{x}_{1}^{\xi}$, P2 takes decision $x_{21}$, and A1 obtains a payoff of $8 \xi+4.5(1-\xi)$. (2) If A1 truthfully reports his type to $\mathrm{P} 1$ and misreports his type to P2, then $\mathrm{P} 1$ implements the lottery $\tilde{x}_{1}^{\xi}$, P2 takes decision $x_{22}$, and A1 obtains a payoff of $\xi+4.5(1-\xi)<8 \xi+4.5(1-\xi)$. (3) If A1 misreports his type to $\mathrm{P} 1$ and truthfully reports his type to $\mathrm{P} 2$, then $\mathrm{P} 1$ implements the lottery $\tilde{x}_{1}^{1-\xi}$, P2 takes decision $x_{12}$, and A1 obtains a payoff of $8(1-\xi)+4.5 \xi \leq 8 \xi+4.5(1-\xi)$ as $\xi \geq \frac{1}{2}$. (4) Finally, if A1 misreports his type to P1 and P2, then P1 implements the lottery $\tilde{x}_{1}^{1-\xi}$, P2 takes decision $x_{22}$, and A1 obtains a payoff of $1-\xi+4.5 \xi<8 \xi+4.5(1-\xi)$. Thus A1 has no incentive to deviate from his candidate equilibrium strategy in state $\left(\omega_{L}, \omega_{L}\right)$, and neither has A2 by symmetry.

Consider next state $\left(\omega_{H}, \omega_{H}\right)$, and suppose that A2 and A3 recommend $d_{1}^{0}$ to P1 and $d_{2}^{*}$ to P2, and that A2 truthfully reports his type to P1 and P2. Then P1 implements the lottery $\tilde{x}_{1}^{1-\xi}$ and $\mathrm{P} 2$ takes decision $x_{22}$ regardless of the reports and/or messages of A1 to $\mathrm{P} 1$ and P2. Thus A1 has no incentive to deviate from his candidate equilibrium strategy in state $\left(\omega_{H}, \omega_{H}\right)$, and neither has A2 by symmetry. This concludes the discussion of Case 1 .

Case 2: $v \in(5.5,6]$ We specify $\tilde{\phi}_{1}^{r}$ as follows. First, we assume that $\bar{\omega}_{1}^{1}=\bar{\omega}_{1}^{2}=\omega_{H}$, so that, if some agent $i=1,2$ sends a message $m_{1}^{i} \notin D_{1} \times \Omega^{i}$ to P1, then $\tilde{\phi}_{1}^{r}$ treats this message as if agent $i$ reported to principal $j$ to be of type $\omega_{H}$; the corresponding property for $\phi_{2}^{r}$ is irrelevant for the following arguments. Fixing some $\xi \in\left[\frac{1}{2}, 1\right]$, we then let

$$
\tilde{d}_{1}(\omega) \equiv \begin{cases}\tilde{x}_{1}^{\xi} & \text { if } \omega=\left(\omega_{H}, \omega_{H}\right) \\ \tilde{x}_{1}^{1-\xi} & \text { otherwise }\end{cases}
$$

where the lotteries $\tilde{x}_{1}^{\xi}$ and $\tilde{x}_{1}^{1-\xi}$ are defined as in Case 1.

We now show that, for each $\xi \in\left(\frac{1}{2}, 1\right]$, the subgame $\left(\tilde{\phi}_{1}^{r}, \phi_{2}^{r}\right)$ admits a BNE in which: (i) each agent recommends to $\mathrm{P} 1$ the direct mechanism $d_{1}^{0}$, and recommends to P2 the direct mechanism $d_{2}^{* *}$ that selects the decision $x_{21}$ regardless of A1's and A2's reports; (b) A1 and A2 truthfully report their types to P1-because P2's decision is fixed, the messages they send to P2 are irrelevant. The corresponding payoff for P2 in the subgame $\left(\tilde{\phi}_{1}^{r}, \phi_{2}^{r}\right)$ is $v=5+\xi \in(5.5,6]$ as $\xi$ varies in $\left(\frac{1}{2}, 1\right]$, as desired. Because A3's payoff is constant over $X \times \Omega$, we only need to focus on A1's and A2's incentives.

Consider first state $\left(\omega_{H}, \omega_{H}\right)$, and suppose that A2 and A3 recommend $d_{1}^{0}$ to P1 and $d_{2}^{* *}$ to $\mathrm{P} 2$, and that A2 truthfully reports his type to P1. Because A1 is not pivotal, recommending a different direct mechanism to either principal is of no avail to him; moreover, because $\bar{\omega}_{1}^{1}=\omega_{H}$, sending a message $m_{1}^{1} \notin D_{1} \times \Omega^{1}$ to P1 amounts for A1 to truthfully reporting his 
type to her. We can thus with no loss of generality assume that A1 recommends $d_{1}^{0}$ to P1 and $d_{2}^{* *}$ to P2, and we only need to study A1's reporting decisions. (1) If A1 truthfully reports his type to $\mathrm{P} 1$, then $\mathrm{P} 1$ implements the lottery $\tilde{x}_{1}^{\xi}$ and $\mathrm{A} 1$ obtains a payoff of $4.5 \xi+1-\xi$.

(2) If A1 misreports his type to P1, then P1 implements the lottery $\tilde{x}_{1}^{1-\xi}$ and A1 obtains a payoff of $4.5(1-\xi)+\xi<4.5 \xi+1-\xi$ as $\xi>\frac{1}{2}$. Thus A1 has no incentive to deviate from his candidate equilibrium strategy in state $\left(\omega_{H}, \omega_{H}\right)$, and neither has A2 by symmetry.

Consider next state $\left(\omega_{L}, \omega_{L}\right)$, and suppose that A2 and A3 recommend $d_{1}^{0}$ to P1 and $d_{2}^{* *}$ to $\mathrm{P} 2$, and that A2 truthfully reports his type to P1. Then P1 implements the lottery $\tilde{x}_{1}^{1-\xi}$ regardless of A1's reports and/or messages to P1. Thus A1 has no incentive to deviate from his candidate equilibrium strategy in state $\left(\omega_{L}, \omega_{L}\right)$, and neither has A2 by symmetry. This concludes the discussion of Case 2.

To conclude the proof, observe that, because P1's payoff is constant over $X \times \Omega$, she has no profitable deviation, and that any deviation by P2 to some arbitrary standard mechanism $\phi_{2}: M_{2} \rightarrow \Delta\left(X_{2}\right)$ can be punished as in Step 1 of the proof, yielding her her minimum feasible payoff of 5 , so that she has no profitable deviation either. The result follows.

\section{References}

[1] Aliprantis, C.D., and K.C. Border (2006): Infinite Dimensional Analysis: A Hitchhiker's Guide. Berlin, Heidelberg, New York: Springer.

[2] Attar, A., E. Campioni, T. Mariotti, and G. Piaser (2021): "Competing Mechanisms and Folk Theorems: Two Examples," Games and Economic Behavior, 125, 79-93.

[3] Attar, A., E. Campioni, and G. Piaser (2019): "Private Communication in Competing Mechanism Games," Journal of Economic Theory, 183, 258-283.

[4] Aumann, R.J. (1961): "Borel Structures for Function Spaces," Illinois Journal of Mathematics, 5(4), 614-630.

[5] Eeckhout, J., and P. Kircher (2010): "Sorting and Decentralized Price Competition," Econometrica, 78(2), 539-574.

[6] Epstein, L.G., and M. Peters (1999): "A Revelation Principle for Competing Mechanisms," Journal of Economic Theory, 88(1), 119-160.

[7] Hurwicz, L. (1973): "The Design of Mechanisms for Resource Allocation," American Economic Review, 63(2), 1-30. 
[8] Kalai A., E. Kalai , E. Lehrer, and D. Samet (2010): "A Commitment Folk Theorem," Games and Economic Behavior, 69(1), 127-137.

[9] Laffont, J.-J., and D. Martimort (1997): "Collusion under Asymmetric Information," Econometrica, 65(4), 875-911.

[10] Martimort, D. and L. Stole (2002): "The Revelation and Delegation Principles in Common Agency Games," Econometrica, 70(4), 1659-1673.

[11] McAfee, R.P. (1993): "Mechanism Design by Competing Sellers," Econometrica, 61(6), 1281-1312.

[12] Milgrom, P.R., and R.J. Weber (1985): "Distributional Strategies for Games with Incomplete Information," Mathematics of Operations Research, 10(4), 619-631.

[13] Moen, E.R. (1997): "Competitive Search Equilibrium," Journal of Political Economy, 105(2), 385-411.

[14] Myerson, R.B. (1979): "Incentive Compatibility and the Bargaining Problem," Econometrica, 47(1), 61-73.

[15] Myerson, R.B. (1982): "Optimal Coordination Mechanisms in Generalized PrincipalAgent Problems," Journal of Mathematical Economics, 10(1), 67-81.

[16] von Negenborn, C., and M. Pollrich (2020): "Sweet Lemons: Mitigating Collusion in Organizations," Journal of Economic Theory, 189(105074), 1-25.

[17] Pavan, A. and G. Calzolari (2009): "Sequential Contracting with Multiple Principals," Journal of Economic Theory, 144(2), 503-531.

[18] Pavan, A. and G. Calzolari (2010): "Truthful Revelation Mechanisms for Simultaneous Common Agency Games," American Economic Journal: Microeconomics, 2(2), 132-190.

[19] Peck, J. (1997): "A Note on Competing Mechanisms and the Revelation Principle," Mimeo, Ohio State University.

[20] Peters, M. (1997): "A Competitive Distribution of Auctions," Review of Economic Studies, 64(1), 97-123.

[21] Peters, M. (2001): "Common Agency and the Revelation Principle," Econometrica, 69(5), 1349-1372. 
[22] Peters, M. (2014): "Competing Mechanisms," Canadian Journal of Economics, 47(2), 373-397.

[23] Peters, M. (2015): "Reciprocal Contracting," Journal of Economic Theory, 158, $102-126$.

[24] Peters, M., and S. Severinov (1997): "Competition among Sellers who Offer Auctions instead of Prices," Journal of Economic Theory, 75(1), 141-179.

[25] Peters, M., and B. Szentes (2012): "Definable and Contractible Contracts," Econometrica, 80(1), 363-411.

[26] Peters, M., and C. Troncoso-Valverde (2013): "A Folk Theorem for Competing Mechanisms," Journal of Economic Theory, 148(3), 953-973.

[27] Prat, A., and A. Rustichini (2003): "Games Played Through Agents," Econometrica, 71(4), 989-1026.

[28] Rao, B.V. (1971): "Borel Structures for Function Spaces," Colloquium Mathematicum, $23(1), 33-38$.

[29] Rahman, D., and I. Obara (2010): "Mediated Partnerships," Econometrica, 78(1), 285-308.

[30] Szentes, B. (2015): "Contractible Contracts in Common Agency Problems," Review of Economic Studies, 82(1), 391-422.

[31] Virág, G. (2010): “Competing Auctions: Finite Markets and Convergence," Theoretical Economics, 5(2), 241-274.

[32] Wright, R., P. Kircher, B. Julien, B., V. Guerrieri (2021): "Directed Search and Competitive Search Equilibrium: A Guided Tour," Journal of Economic Literature, $59(1), 90-148$.

[33] Xiong, S. (2013): "A Folk Theorem for Contract Games with Multiple Principals and Agents," Mimeo, Departement of Economics, Rice University.

[34] Yamashita, T. (2010): "Mechanism Games With Multiple Principals and Three or More Agents," Econometrica, 78(2), 791-801. 